University of Nebraska - Lincoln

DigitalCommons@University of Nebraska - Lincoln

Roman L. Hruska U.S. Meat Animal Research

U.S. Department of Agriculture: Agricultural Center

Research Service, Lincoln, Nebraska

2010

\title{
REVIEW: Preweaning, Postweaning, and Carcass Trait Comparisons for Progeny Sired by Subtropically Adapted Beef Sire Breeds at Various US Locations
}

F. A. Thrift

University of Kentucky, Lexington

J. O. Sanders

Texas A\&M University, College Station

M. A. Brown

USDA-ARS, Grazinglands Research Laboratory, EI Reno, OK

A. H. Brown Jr

University of Arkansas, Fayetteville

A. D. Herring

USDA-ARS, Grazinglands Research Laboratory, EI Reno, OK

See next page for additional authors

Follow this and additional works at: https://digitalcommons.unl.edu/hruskareports

Part of the Animal Sciences Commons

Thrift, F. A.; Sanders, J. O.; Brown, M. A.; Brown Jr, A. H.; Herring, A. D.; Riley, D. G.; DeRouen, S. M.; Holloway, J. W.; Wyatt, W. E.; Vann, R. C.; Chase, C. C. Jr.; Franke, D. E.; Cundiff, L. V.; and Baker, J. F., "REVIEW: Preweaning, Postweaning, and Carcass Trait Comparisons for Progeny Sired by Subtropically Adapted Beef Sire Breeds at Various US Locations" (2010). Roman L. Hruska U.S. Meat Animal Research Center. 166.

https://digitalcommons.unl.edu/hruskareports/166

This Article is brought to you for free and open access by the U.S. Department of Agriculture: Agricultural Research Service, Lincoln, Nebraska at DigitalCommons@University of Nebraska - Lincoln. It has been accepted for inclusion in Roman L. Hruska U.S. Meat Animal Research Center by an authorized administrator of DigitalCommons@University of Nebraska - Lincoln. 


\section{Authors}

F. A. Thrift, J. O. Sanders, M. A. Brown, A. H. Brown Jr, A. D. Herring, D. G. Riley, S. M. DeRouen, J. W. Holloway, W. E. Wyatt, R. C. Vann, C. C. Chase Jr., D. E. Franke, L. V. Cundiff, and J. F. Baker 


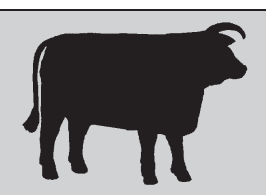

$R_{\text {EVIEW: Preweaning, }}$

Postweaning, and Carcass

Trait Comparisons for Progeny

Sired by Subtropically Adapted

Beef Sire Breeds at Various US

Locations 1,2,3 $^{1,3}$

F. A. Thrift*4 PAS, J O Sanders $t$ M. A. Brown $\neq$ PAS, A. H. Brown Jr § PAS, A. D. Herring PAS, D. G. Riley,† S. M. DeRouen,\# PAS, J. W. Holloway, „ W. E. Wyatt, \ PAS, R. C. Vann, ${ }^{* *}$ PAS, C. C. Chase Jr., †† D. E. Franke, ¥¥ PAS, L. V. Cundiff,§§ PAS, and J. F. Baker,\#\# PAS *Department of Animal and Food Sciences, University of Kentucky, Lexington 40546; †Department of Animal Science, Texas A\&M University, College Station 77843; łUSDA-ARS, Grazinglands Research Laboratory, El Reno, OK 73036; §Department of Animal Sciences, University of Arkansas, Fayetteville 72701; \#Dean Lee Research Station, Alexandria, LA 71302; IITexas Agricultural Experiment Station, Uvalde 78801; T|beria Research Station, Jeanerette, LA 70544; **Brown Loam Experiment Station, Raymond, MS 39154; ††USDA-ARS, Subtropical Agricultural Research Station, Brooksville, FL 34601; $¥ \ddagger$ Department of Animal Science, Louisiana State University Agricultural Center, Baton Rouge 70803; §§USDA-ARS, US Meat Animal Research Center, Clay Center, NE 68933; and \#\#Department of Animal and Dairy Science, University of Georgia, Tifton 31793

${ }^{1}$ This review represents the summarization of results generated at locations that participated in Hatch Act multistate beef cattle genetics research projects S-243 [Evaluation of Beef Cattle Germplasm Resources Involving Additive and NonAdditive Genetic Effects], S-277 [Breeding to Optimize Maternal Performance and Reproduction of Beef Cows in the Southern Region], and S-1013 [Genetic (Co)variance of Parasite Resistance, Temperament and Production Traits of Traditional and Non-Bos indicus Tropical Adapted Breeds].

\section{ABSTRACT}

This review, which represents a summarization of research results generated during an approximately 22-yr period,

\footnotetext{
${ }^{2}$ Mention of a trade name, proprietary product, or specific equipment does not constitute a guarantee or warranty by the USDA and does not imply approval to the exclusion of other products that may be suitable.

${ }^{3}$ Published with approval of the director, Kentucky Agricultural Experiment Station (Lexington) as Journal Article 09-07-090. ${ }^{4}$ Corresponding author: fthrift@uky.edu
}

involves preweaning, postweaning, and carcass trait comparisons of progeny sired by Bos indicus (Brahman, Boran, Nellore, Indu-Brazil, Gir, Sahiwal), B. indicus-derivative (Brangus, Beefmaster, Santa Gertrudis, Gelbray, Simbrah), non-B. indicus (Tuli, Romosinuano, Bonsmara, Senepol) subtropically adapted, and traditional Bos taurus (Angus, Hereford, Charolais, Gelbvieh, Red Poll) sire breeds. Relative to Brahman-sired progeny, preweaning (weaning weight) and postweaning (postweaning $A D G$, feedlot $A D G$, final feedlot $B W$ ) performance is expected to be less for progeny 
sired by non-B. indicus subtropically adapted sire breeds. The non-B. indicus subtropically adapted sire breeds do contribute to less dystocia and appear to improve carcass merit, especially carcass tenderness, over the Brahman breed. Other B. indicus sire breeds, such as the Gir and Sahiwal, but not the Indu-Brazil, contribute to less dystocia compared with the Brahman breed. Relative to B. indicus and non-B. indicus subtropically adapted sire breeds, B. taurus sire breeds, especially Angus and Hereford, express superior carcass merit in regard to marbling score, $Q G$, and tenderness.

Key words: beef cattle, subtropical adaptation, Bos indicus, Bos taurus

\section{INTRODUCTION}

Considerable effort has been directed toward evaluating the Brahman breed, primarily for crossbreeding, in a wide array of environments. Results indicate that in the hot, humid Southeast and Gulf Coast areas, and even in more temperate areas of the United States, the weaning productivity of Brahman $\times$ Bos taurus cows is virtually unequaled; however, problems have been identified with the Brahman breed. Specifically, 1) subpar reproductive performance of Brahman bulls, 2) increased dystocia or reduced survival rate expressed by Brahmansired calves, 3) price discounts for Brahman-sired steers and surplus heifers contemporary to Brahman-sired replacement heifers, 4) unfavorable carcass attributes, especially tenderness, and 5) nondocile temperament expressed by Brahman-influenced cattle are problems associated with the Brahman breed that have served as a major impetus to evaluate alternative sources of subtropically adapted beef cattle germplasm at locations primarily in the Southeast and Gulf Coast areas (Thrift and Thrift, 2005).

\section{MATERIALS AND METHODS}

This review represents a summarization of comparisons involving preweaning, postweaning, and carcass traits of progeny sired by Bos indicus
(Brahman, Boran, Nellore, InduBrazil, Gir, Sahiwal), B. indicus-derivative (Brangus, Beefmaster, Santa Gertrudis, Gelbray, Simbrah), non- $B$. indicus (Tuli, Romosinuano, Bonsmara, Senepol) subtropically adapted sire breeds, and traditional B. taurus (Angus, Hereford, Charolais, Gelbvieh, Red Poll) sire breeds.

Because of its wide-scale usage in the Southeast and Gulf Coast areas, the Brahman was the primary sire breed with which most other sire breeds were compared. When available for specific traits, the sire breed comparisons summarized were as follows: 1) Brahman versus traditional B. taurus sire breeds; 2) Brahman versus other $B$. indicus sire breeds; 3 ) Brahman versus $B$. indicus-derivative sire breeds; 4) Brahman versus non- $B$. indicus subtropically adapted sire breeds; 5) B. indicus-derivative versus traditional $B$. taurus sire breeds; 6$) B$. indicus-derivative versus non- $B$. indicus subtropically adapted sire breeds; and 7) non- $B$. indicus subtropically adapted versus traditional $B$. taurus sire breeds.

Where available for each study, sire breed comparisons were summarized for preweaning (percentage of unassisted births, gestation length, birth weight, weaning weight), postweaning (postweaning $\mathrm{ADG}$, feedlot $\mathrm{ADG}$, final feedlot BW), and carcass (hot carcass weight, YG, QG, marbling score, Warner-Bratzler shear force value, LM area) traits.

Most studies summarized contributed to at least 1 of 3 multistate beef cattle genetic research projects (S243, S-277, S-1013) that collectively spanned an approximately $22-y r$ period. Although not contributing to the multistate research projects, results were also summarized from 3 early studies (Gregory et al., 1979; Koch et al., 1982; Crouse et al., 1989) that evaluated 2 subtropically adapted sire breeds in Cycle III of the MARC Germplasm Evaluation Program (Clay Center, NE).

Most sire breed comparisons summarized involved mating schemes resulting in production of either 2- or 3 -breed-cross progeny that express
$100 \%$ direct heterosis; however, some sire breed comparisons involved straightbred and crossbred matings (Brown et al., 1993a,b; Chase et al., 1998; Riley et al., 2007) and some sire breed comparisons involved only straightbred matings (DeRouen et al., 1992; Thrift et al., 1999; Bidner et al., 2002; Phillips et al., 2006). As indicated by Paschal et al. (1991), in studies in which sires of 2 (or more) different breeds are mated to dams of a third genetic type, sire breed differences represent one-half the difference in direct breed effects plus differences attributed to direct heterosis of the different sire breeds with the dam genetic type.

Typically, for studies that involved crossbreeding, it was assumed that direct heterosis was the same for all breed crosses. This assumption is probably valid when different $B$. indicus or different B. taurus sire breeds are compared. However, in situations in which $B$. indicus (or $B$. indicus-derivative) and B. taurus sire breeds are compared when mated to dams of a third genetic type, the assumption that the $B$. indicus (or $B$. indicus-derivative) $\times B$. taurus and $B$. taurus $\times B$. taurus crosses express equal direct heterosis probably is not valid, especially for preweaning traits (Franke, 1980; Gregory and Cundiff, 1980; Koger, 1980; Long, 1980). Thus, sire breed differences when $B$. indicus (or $B$. indicus-derivative) versus $B$. taurus or when $B$. indicus (or $B$. indicus-derivative) versus non- $B$. indicus subtropically adapted sire breeds are involved should be interpreted with this in mind.

\section{RESULTS AND DISCUSSION}

\section{Preweaning Traits}

Preweaning trait (percentage of unassisted calvings, gestation length, birth weight, weaning weight) comparisons involving progeny sired by subtropically adapted beef sire breeds are summarized in Table 1. Results are summarized by comparing sire breed means for each trait. 
Table 1. Preweaning trait comparisons involving progeny sired by subtropically adapted beef sire breeds

Trait $^{1}$

\begin{tabular}{|c|c|c|c|c|c|c|c|}
\hline Sire breed comparison & $\mathbf{N}$ & $\%$ UC & GL & $\mathbf{N}$ & Birth wt, kg & $\mathbf{N}$ & Weaning wt, kg \\
\hline \multicolumn{8}{|c|}{ Brahman vs. traditional Bos taurus sire breeds } \\
\hline \multicolumn{8}{|c|}{ Gregory et al. (1979) } \\
\hline Brahman & 349 & 87.0 & 349291.9 & 349 & 40.6 & 330 & 215.0 \\
\hline Angus/Hereford & 358 & 94.1 & 358284.2 & 358 & 35.4 & 346 & 203.0 \\
\hline Difference & & $-7.1^{* *}$ & $7.7^{\star *}$ & & $5.2^{\star *}$ & & $12.0^{* *}$ \\
\hline \multicolumn{8}{|l|}{ Roberson et al. (1986) } \\
\hline Brahman & & & & 1,387 & 34.1 & 1,191 & 146.8 \\
\hline Hereford & & & & 2,085 & 31.8 & 1,742 & 155.6 \\
\hline Difference & & & & & $2.3^{* *}$ & & $-8.8^{* *}$ \\
\hline \multicolumn{8}{|l|}{ Sanders et al. $(1987)^{2}$} \\
\hline Zebu & & & 250289.6 & 249 & 34.7 & 224 & 210.2 \\
\hline Angus & & & 94281.3 & 93 & 30.3 & 85 & 198.8 \\
\hline Difference & & & $8.3 \mathrm{NT}$ & & $4.4 \mathrm{NT}$ & & $11.4 \mathrm{NT}$ \\
\hline \multicolumn{8}{|l|}{ Neville et al. (1988) } \\
\hline Brahman & & & & 94 & 33.6 & 94 & 192.0 \\
\hline Angus & & & & 88 & 30.1 & 88 & 177.0 \\
\hline Difference & & & & & $3.5^{\star *}$ & & $15.0^{* *}$ \\
\hline \multicolumn{8}{|l|}{ Paschal et al. (1991) } \\
\hline Gray Brahman & 48 & 87.0 & 48291.0 & 48 & 37.1 & 44 & 212.9 \\
\hline Angus & 40 & 95.0 & 40282.0 & 39 & 31.8 & 36 & 198.7 \\
\hline Difference & & $-8.0^{*}$ & $9.0^{*}$ & & $5.3^{*}$ & & $14.2^{*}$ \\
\hline \multicolumn{8}{|l|}{ Brown et al. (1993b) } \\
\hline Brahman & & & & 213 & 36.9 & 193 & 202.2 \\
\hline Angus & & & & 273 & 33.1 & 261 & 198.4 \\
\hline Difference & & & & & $3.8^{* *}$ & & $3.8 \mathrm{NS}$ \\
\hline \multicolumn{8}{|l|}{ Browning et al. (1995) } \\
\hline Brahman & & & 99293.7 & 99 & 31.2 & 99 & 198.8 \\
\hline Angus & & & 68284.0 & 68 & 30.3 & 68 & 220.9 \\
\hline Difference & & & $9.7^{* *}$ & & 0.9 NS & & $-22.1^{\star *}$ \\
\hline \multicolumn{8}{|l|}{ Thrift et al. (1999) } \\
\hline Brahman & & & & 889 & 27.7 & 786 & 191.5 \\
\hline Angus & & & & 2,365 & 25.0 & 2,274 & 174.0 \\
\hline Difference & & & & & $2.7^{*}$ & & $17.5^{*}$ \\
\hline \multicolumn{8}{|l|}{ Cundiff (2005) } \\
\hline Brahman & 436 & 89.4 & 436291.4 & 436 & 44.3 & 436 & 245.9 \\
\hline Angus & 1,021 & 97.6 & $1,021282.5$ & 1,021 & 38.7 & 1,021 & 240.0 \\
\hline Difference & & $-8.2^{*}$ & $8.9^{*}$ & & $5.6^{*}$ & & $5.9^{*}$ \\
\hline \multicolumn{8}{|l|}{ Riley et al. (2007) } \\
\hline Brahman & & & & 427 & 34.3 & 394 & 229.6 \\
\hline Angus & & & & 424 & 30.8 & 316 & 224.0 \\
\hline Difference & & & & & $3.5^{\star *}$ & & $5.6^{* *}$ \\
\hline \multicolumn{8}{|l|}{ Amen et al. (2007a) } \\
\hline Brahman & & & 141290.5 & 141 & 38.4 & 134 & 236.1 \\
\hline Angus & & & 137282.7 & 139 & 34.3 & 132 & 229.8 \\
\hline Difference & & & $7.8^{*}$ & & 4.1 NS & & $6.3 \mathrm{NS}$ \\
\hline \multicolumn{8}{|l|}{ Thrift et al. (1999) } \\
\hline Brahman & & & & 718 & 28.9 & 550 & 162.5 \\
\hline Hereford & & & & 2,276 & 30.4 & 2,107 & 161.0 \\
\hline Difference & & & & & $-1.5 \mathrm{NS}$ & & $1.5 \mathrm{NS}$ \\
\hline \multicolumn{8}{|l|}{ Baker et al. (2001a) } \\
\hline Brahman & & & & & 38.4 & & 250.7 \\
\hline Hereford & & & & & 34.5 & & 232.2 \\
\hline Difference & & & & & $3.9 \mathrm{NS}$ & & $18.5 \mathrm{NS}$ \\
\hline \multicolumn{8}{|l|}{ Cundiff (2005) } \\
\hline Brahman & 436 & 89.4 & 436291.4 & 436 & 44.3 & 436 & 245.9 \\
\hline
\end{tabular}


Table 1 (Continued). Preweaning trait comparisons involving progeny sired by subtropically adapted beef sire breeds

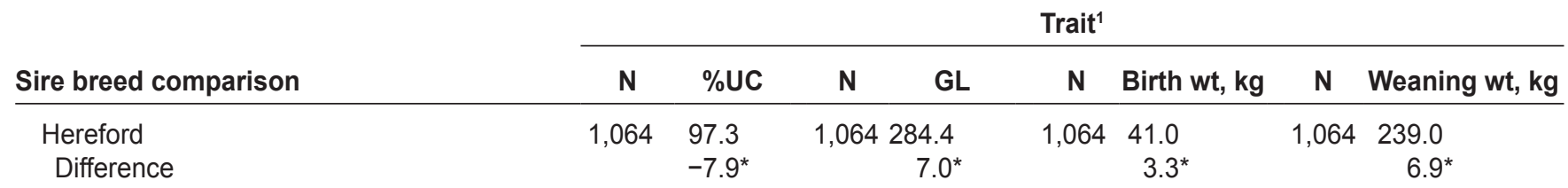

Brahman vs. other Bos indicus sire breeds

Gregory et al. (1979)

\section{Brahman \\ Sahiwal}

Difference

Paschal et al. (1991)

Gray Brahman

Red Brahman

Difference

Paschal et al. (1991)

Gray Brahman

Gir

Difference

Paschal et al. (1991)

Gray Brahman

Indu-Brazil

Difference

Paschal et al. (1991)

Gray Brahman

Nellore

Difference

Cundiff (2005)

Brahman

Nellore

Difference

Herring et al. (1996)

Brahman

Boran

Difference

Cundiff (2005)

Brahman

Boran

Difference

Brahman vs. B. indicus-derivative sire breeds

Cundiff (2005)

Brahman

Brangus

Difference

Brown et al. (1997)

Brahman

Beefmaster

Difference

Cundiff (2005)

Brahman

Beefmaster

Difference

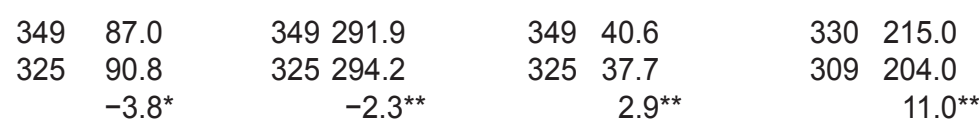

$\begin{array}{llllllll}48 & 87.0 & 48 & 291.0 & 48 & 37.1 & 44 & 212.9\end{array}$

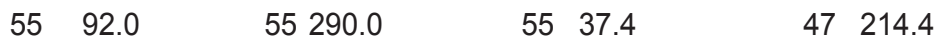

$-5.0 \mathrm{NS} \quad 1.0 \mathrm{NS} \quad-0.3 \mathrm{NS}$

-1.5 NS

$\begin{array}{lll}48 & 87.0 \quad 48291.0\end{array}$

49289.0

$2.0 \mathrm{NS}$

$48 \quad 37.1$

4933.0

$4.1^{*}$

$44 \quad 212.9$

$\begin{array}{ll}45 & 197.8\end{array}$

$15.1^{*}$

$\begin{array}{ll}48 & 87.0\end{array}$

48291.0

47290.0

$1.0 \mathrm{NS}$

$\begin{array}{ll}48 & 37.1\end{array}$

$47 \quad 39.1$

$-2.0^{*}$

$44 \quad 212.9$

$42 \quad 205.1$

$7.8 \mathrm{NS}$

$48 \quad 87.0$

48291.0

51294.0

86.0

$-3.0^{*}$

$\begin{array}{ll}48 & 37.1\end{array}$

$44 \quad 212.9$

$\begin{array}{ll}50 & 36.7\end{array}$

0.4 NS

$46 \quad 206.3$

6.6 NS

\section{$\begin{array}{lll}436 & 89.4 & 436291.4\end{array}$}

$196 \quad 94.6$

$-5.2^{*}$

196292.9

-1.5 NS

$436 \quad 44.3$

$196 \quad 42.7$

$1.6^{*}$

$\begin{array}{ll}436 & 245.9\end{array}$

$196 \quad 243.1$

101288.6

104289.7

$-1.1 \mathrm{NS}$

10144.0

$103 \quad 40.3$

$3.7^{*}$

$97 \quad 234.3$

$95 \quad 217.1$

$17.2^{*}$

$\begin{array}{lll}436 & 89.4 & 436291.4\end{array}$

$456 \quad 94.1$

$-4.7^{*}$

456291.2

0.2 NS

$436 \quad 44.3$

$456 \quad 41.2$

$3.1^{*}$

$436 \quad 245.9$

$456 \quad 229.1$

$16.8^{*}$
$436 \quad 44.3$

$214 \quad 40.4$

$3.9^{*}$

$57 \quad 41.0$

$30 \quad 43.0$

$-2.0 \mathrm{NS}$

$436 \quad 89.4$

222
96.1

$-6.7^{*}$
436291.4

222287.4

$4.0^{*}$
$436 \quad 44.3$

$222 \quad 42.7$

$1.6^{*}$ $\begin{array}{ll}436 & 245.9\end{array}$

$214 \quad 242.2$

$3.7 \mathrm{NS}$

$\begin{array}{ll}55 & 235.0\end{array}$

$27 \quad 238.0$

-3.0 NS

$\begin{array}{ll}436 & 245.9\end{array}$

$\begin{array}{ll}222 & 247.7\end{array}$

-1.8 NS

Continued 
Table 1 (Continued). Preweaning trait comparisons involving progeny sired by subtropically adapted beef sire breeds

Trait $^{1}$

Sire breed comparison

\begin{tabular}{llllllll}
\hline $\mathrm{N}$ & $\% \mathrm{UC}$ & $\mathrm{N}$ & $\mathrm{GL}$ & $\mathrm{N}$ & Birth wt, kg & $\mathrm{N}$ & Weaning wt, kg \\
\hline
\end{tabular}

Brahman vs. non- $B$. indicus subtropically adapted sire breeds

Browning et al. (1995)

Brahman

Tuli

Difference

Herring et al. (1996)

Brahman

Tuli

Difference

Chase et al. (2000)

Brahman

Tuli

Difference

Baker et al. (2001a)

Brahman

Tuli

Difference

Holloway et al. (2002)

Brahman

Tuli

Difference

Cundiff (2005)

Brahman

Tuli

Difference

Cundiff (2005)

Brahman

Romosinuano

Difference

Riley et al. (2007)

Brahman

Romosinuano

Difference

Cundiff (2005)

Brahman

Bonsmara

Difference

Sanders et al. (1987)

Zebu

Senepol

Difference

Chase et al. (2000)

Brahman

Senopol

Difference

Baker et al. (2001a)

Brahman

Senepol

Difference

Holloway et al. (2002)

Brahman

Senepol

Difference

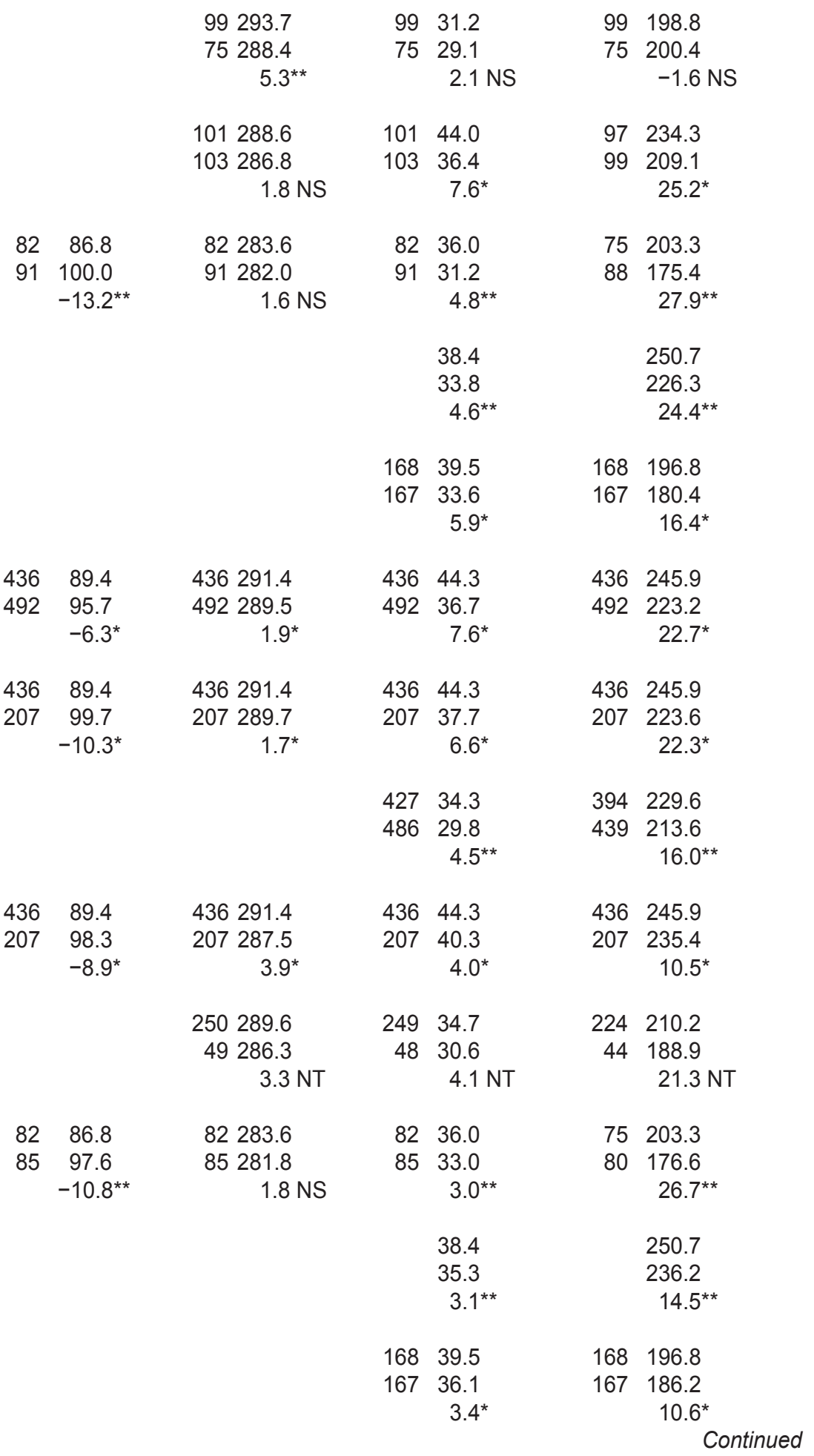


Table 1 (Continued). Preweaning trait comparisons involving progeny sired by subtropically adapted beef sire breeds

\begin{tabular}{|c|c|c|c|c|c|c|c|}
\hline \multirow[b]{2}{*}{ Sire breed comparison } & \multicolumn{7}{|c|}{ Trait $^{1}$} \\
\hline & $\mathbf{N}$ & $\% U C$ & GL & $\mathbf{N}$ & Birth wt, kg & $\mathbf{N}$ & Weaning w \\
\hline \multicolumn{8}{|c|}{$\begin{array}{l}\text { B. indicus-derivative vs. traditional B. taurus sire } \\
\text { breeds }\end{array}$} \\
\hline \multicolumn{8}{|c|}{ Wheeler et al. (2006) } \\
\hline Brangus & 214 & 96.9 & 214284.9 & 214 & 41.1 & 214 & 249.0 \\
\hline Angus & 208 & 97.2 & 208281.6 & 208 & 39.5 & 208 & 245.4 \\
\hline Difference & & $-0.3 \mathrm{NS}$ & $3.3^{*}$ & & $1.6^{*}$ & & $3.6 \mathrm{NS}$ \\
\hline \multicolumn{8}{|l|}{ Wheeler et al. (2006) } \\
\hline Brangus & 214 & 96.9 & 214284.9 & 214 & 41.1 & 214 & 249.0 \\
\hline Hereford & 212 & 94.4 & 212283.7 & 212 & 41.3 & 212 & 242.2 \\
\hline Difference & & $2.5 \mathrm{NS}$ & $1.2 \mathrm{NS}$ & & $-0.2 \mathrm{NS}$ & & $6.8^{*}$ \\
\hline \multicolumn{8}{|l|}{ Wheeler et al. (2006) } \\
\hline Beefmaster & 222 & 95.6 & 222286.6 & 222 & 43.3 & 222 & 254.0 \\
\hline Angus & 208 & 97.2 & 208281.6 & 208 & 39.5 & 208 & 245.4 \\
\hline Difference & & $-1.6 \mathrm{NS}$ & $5.0^{*}$ & & $3.8^{*}$ & & $8.6^{*}$ \\
\hline \multicolumn{8}{|l|}{ Wheeler et al. (2006) } \\
\hline Beefmaster & 222 & 95.6 & 222286.6 & 222 & 43.3 & 222 & 254.0 \\
\hline Hereford & 212 & 94.4 & 212283.7 & 212 & 41.3 & 212 & 242.2 \\
\hline Difference & & 1.2 NS & $2.9^{*}$ & & $2.0^{*}$ & & $11.8^{*}$ \\
\hline \multicolumn{8}{|l|}{ Oxford et al. (2006) } \\
\hline Santa Gertrudis & & & & 103 & 32.8 & 103 & 192.5 \\
\hline Angus & & & & 109 & 27.3 & 109 & 184.2 \\
\hline Difference & & & & & $5.5^{*}$ & & $8.3^{*}$ \\
\hline \multicolumn{8}{|l|}{ Oxford et al. (2006) } \\
\hline Santa Gertrudis & & & & 123 & 31.2 & 123 & 215.4 \\
\hline Hereford & & & & 130 & 29.8 & 130 & 196.0 \\
\hline Difference & & & & & $1.4^{*}$ & & $19.4^{* *}$ \\
\hline \multicolumn{8}{|l|}{ Oxford et al. (2006) } \\
\hline Santa Gertrudis & & & & 226 & 32.0 & 226 & 204.0 \\
\hline Charolais & & & & 162 & 32.1 & 162 & 206.5 \\
\hline Difference & & & & & $-0.1 \mathrm{NS}$ & & $-2.5 \mathrm{NS}$ \\
\hline \multicolumn{8}{|l|}{ Oxford et al. (2006) } \\
\hline Santa Gertrudis & & & & 226 & 32.0 & 226 & 204.0 \\
\hline Red Poll & & & & 244 & 27.6 & 244 & 191.9 \\
\hline Difference & & & & & $4.4^{*}$ & & $12.1^{* *}$ \\
\hline
\end{tabular}

Non- $B$. indicus subtropically adapted vs. traditional $B$. taurus sire breeds

Browning et al. (1995)

Tuli

Angus

Difference

Baker et al. (2001a)

Tuli

Angus

Difference

Cundiff (2005)

Tuli

Angus

Difference

Cundiff (2005)

Tuli

Hereford

Difference

Baker et al. (2001a)

\begin{tabular}{|c|c|c|c|c|}
\hline 75288.4 & 75 & 29.1 & 75 & 200.4 \\
\hline 68284.0 & 68 & 30.3 & 68 & 220.9 \\
\hline \multirow[t]{4}{*}{$4.4^{* *}$} & & -1.2 NS & & $-20.5^{\star \star}$ \\
\hline & & 32.8 & & 226.1 \\
\hline & & 32.5 & & 239.8 \\
\hline & & $0.3 \mathrm{NS}$ & & $-13.7^{* *}$ \\
\hline 492289.5 & 492 & 36.7 & 492 & 223.2 \\
\hline 021282.5 & 1,021 & 38.7 & 1,021 & 240.0 \\
\hline $7.0^{*}$ & & $-2.0^{*}$ & & $-16.8^{*}$ \\
\hline 492289.5 & 492 & 36.7 & 492 & 223.2 \\
\hline 064284.4 & 1,064 & 41.0 & 1,064 & 239.0 \\
\hline $5.1^{*}$ & & $-4.3^{*}$ & & $-15.8^{*}$ \\
\hline
\end{tabular}


Table 1 (Continued). Preweaning trait comparisons involving progeny sired by subtropically adapted beef sire breeds

\begin{tabular}{|c|c|c|c|c|c|c|c|}
\hline Sire breed comparison & \multicolumn{7}{|c|}{ Trait $^{1}$} \\
\hline Tuli & & & & & 32.8 & & 226.1 \\
\hline Difference & & & & & $-1.5 \mathrm{NS}$ & & $-17.2^{\star *}$ \\
\hline \multicolumn{8}{|l|}{ Riley et al. (2007) } \\
\hline Romosinuano & & & & 486 & 29.8 & 439 & 213.6 \\
\hline Difference & & & & & $-1.0^{* *}$ & & $-10.4^{* *}$ \\
\hline \multicolumn{8}{|l|}{ Cundiff (2005) } \\
\hline Romosinuano & 207 & 99.7 & 207289.7 & 207 & 37.7 & 207 & 223.6 \\
\hline Angus & 1,021 & 97.6 & $1,021282.5$ & 1,021 & 38.7 & 1,021 & 240.0 \\
\hline Difference & & $2.1 \mathrm{NS}$ & $7.2^{*}$ & & $-1.0^{*}$ & & $-16.4^{*}$ \\
\hline \multicolumn{8}{|l|}{ Wheeler et al. (2006) } \\
\hline \multicolumn{8}{|l|}{ Cundiff (2005) } \\
\hline Romosinuano & 207 & 99.7 & 207289.7 & 207 & 37.7 & 207 & 223.6 \\
\hline Hereford & 1,064 & 97.3 & $1,064284.4$ & 1,064 & 41.0 & 1,064 & 239.0 \\
\hline Difference & & $2.4 \mathrm{NS}$ & $5.3^{*}$ & & $-3.3^{*}$ & & $-15.4^{*}$ \\
\hline \multicolumn{8}{|l|}{ Wheeler et al. (2006) } \\
\hline Romosinuano & 207 & 99.2 & 207288.9 & 207 & 38.4 & 207 & 230.0 \\
\hline Hereford & 212 & 94.4 & 212283.7 & 212 & 41.3 & 212 & 242.2 \\
\hline Difference & & $4.8^{*}$ & $5.2^{*}$ & & $-2.9^{*}$ & & $-12.2^{*}$ \\
\hline \multicolumn{8}{|l|}{ Brown and Lalman (2008) } \\
\hline Romosinuano & & & & 57 & 39.0 & 55 & 224.0 \\
\hline Hereford & & & & 70 & 41.0 & 64 & 234.0 \\
\hline Difference & & & & & $-2.0^{*}$ & & $-10.0^{*}$ \\
\hline \multicolumn{8}{|l|}{ Cundiff (2005) } \\
\hline Bonsmara & 207 & 98.3 & 207287.5 & 207 & 40.3 & 207 & 235.4 \\
\hline Angus & 1,021 & 97.6 & $1,021282.5$ & 1,021 & 38.7 & 1,021 & 240.0 \\
\hline Difference & & $0.7 \mathrm{NS}$ & $5.0^{*}$ & & $1.6^{*}$ & & $-4.6 \mathrm{NS}$ \\
\hline \multicolumn{8}{|l|}{ Wheeler et al. (2006) } \\
\hline Bonsmara & 207 & 97.7 & 207286.7 & 207 & 41.0 & 207 & 241.8 \\
\hline Angus & 208 & 97.2 & 208281.6 & 208 & 39.5 & 208 & 245.4 \\
\hline Difference & & $0.5 \mathrm{NS}$ & $5.1^{*}$ & & $1.5^{*}$ & & $-3.6 \mathrm{NS}$ \\
\hline \multicolumn{8}{|l|}{ Cundiff (2005) } \\
\hline Bonsmara & 207 & 98.3 & 207287.5 & 207 & 40.3 & 207 & 235.4 \\
\hline Hereford & 1,064 & 97.3 & $1,064284.4$ & 1,064 & 41.0 & 1,064 & 239.0 \\
\hline Difference & & $1.0 \mathrm{NS}$ & $3.1^{*}$ & & $-0.7 \mathrm{NS}$ & & $-3.6 \mathrm{NS}$ \\
\hline \multicolumn{8}{|l|}{ Wheeler et al. (2006) } \\
\hline Bonsmara & 207 & 97.7 & 207286.7 & 207 & 41.0 & 207 & 241.8 \\
\hline Hereford & 212 & 94.4 & 212283.7 & 212 & 41.3 & 212 & 242.2 \\
\hline Difference & & $3.3 \mathrm{NS}$ & $3.0^{*}$ & & $-0.3 \mathrm{NS}$ & & -0.4 NS \\
\hline \multicolumn{8}{|l|}{ Brown and Lalman (2008) } \\
\hline Bonsmara & & & & 91 & 41.0 & 86 & 236.0 \\
\hline \multirow[t]{2}{*}{ Hereford } & & & & 70 & 41.0 & 64 & 234.0 \\
\hline & & & & & & & Continued \\
\hline
\end{tabular}




\section{Table 1 (Continued). Preweaning trait comparisons involving progeny sired by subtropically adapted beef sire breeds}

\begin{tabular}{|c|c|c|c|c|c|c|}
\hline \multirow[b]{2}{*}{ Sire breed comparison } & \multicolumn{6}{|c|}{ Trait $^{1}$} \\
\hline & $\% U C$ & GL & $\mathbf{N}$ & Birth wt, kg & $\mathbf{N}$ & Weaning wt, kg \\
\hline Difference & & & & $0.0 \mathrm{NS}$ & & $2.0 \mathrm{NS}$ \\
\hline \multicolumn{7}{|l|}{ Brown and Lalman (2008) } \\
\hline Bonsmara & & & 91 & 41.0 & 86 & 236.0 \\
\hline Gelbvieh & & & 68 & 42.0 & 67 & 241.0 \\
\hline Difference & & & & $-1.0 \mathrm{NS}$ & & $-5.0 \mathrm{NS}$ \\
\hline \multicolumn{7}{|l|}{ Brown and Lalman (2008) } \\
\hline Bonsmara & & & 91 & 41.0 & 86 & 236.0 \\
\hline Charolais & & & 68 & 45.0 & 62 & 247.0 \\
\hline Difference & & & & $-4.0^{*}$ & & $-11.0^{*}$ \\
\hline \multicolumn{7}{|l|}{ Sanders et al. (1987) } \\
\hline Senepol & & 49286.3 & 48 & 30.6 & 44 & 188.9 \\
\hline Angus & & 94281.3 & 93 & 30.3 & 85 & 198.8 \\
\hline Difference & & $5.0 \mathrm{NT}$ & & $0.3 \mathrm{NT}$ & & -9.9 NT \\
\hline \multicolumn{7}{|l|}{ Baker et al. (2001a) } \\
\hline Senepol & & & & 34.5 & & 238.6 \\
\hline Angus & & & & 32.5 & & 239.8 \\
\hline Difference & & & & $2.0 \mathrm{NS}$ & & $-1.2 \mathrm{NS}$ \\
\hline \multicolumn{7}{|c|}{ Thrift et al. (1986), Kentucky study } \\
\hline Senepol & & & 119 & 34.3 & 113 & 182.5 \\
\hline Hereford & & & 83 & 33.0 & 79 & 179.4 \\
\hline Difference & & & & $1.3^{*}$ & & $3.1 \mathrm{NS}$ \\
\hline \multicolumn{7}{|c|}{ Chase et al. (1998), phase I } \\
\hline Senepol & & & 194 & 34.1 & 194 & 214.0 \\
\hline Hereford & & & 383 & 32.1 & 383 & 183.0 \\
\hline Difference & & & & $2.0^{* *}$ & & $31.0^{* *}$ \\
\hline \multicolumn{7}{|c|}{ Chase et al. (1998), phase II } \\
\hline Senepol & & & 174 & 34.0 & 174 & 212.0 \\
\hline Hereford & & & 204 & 33.5 & 204 & 211.5 \\
\hline Difference & & & & $0.5 \mathrm{NS}$ & & $0.5 \mathrm{NS}$ \\
\hline \multicolumn{7}{|l|}{ Baker et al. (2001a) } \\
\hline Senepol & & & & 34.5 & & 238.6 \\
\hline Hereford & & & & 34.3 & & 243.3 \\
\hline Difference & & & & $0.2 \mathrm{NS}$ & & $-4.7 \mathrm{NS}$ \\
\hline \multicolumn{7}{|c|}{ Thrift et al. (1986), Louisiana study } \\
\hline Senepol & & & 150 & 28.1 & 136 & 196.0 \\
\hline Red Poll & & & 163 & 29.3 & 146 & 208.0 \\
\hline Difference & & & & $-1.2^{* *}$ & & $-12.0^{* *}$ \\
\hline
\end{tabular}

$1 \% \mathrm{UC}=$ percentage of unassisted calvings; $\mathrm{GL}=$ gestation length .

${ }^{2}$ Zebu collectively refers to average for Gray Brahman, Red Brahman, Gir, Indu-Brazil, and Nellore.

${ }^{* *} P<0.01$; ${ }^{*} P<0.05$; NS = not significant $(P>0.10)$; NT = not tested.

\section{Brahman Versus Traditional} Bos taurus Sire Breeds. Relative to Angus and Hereford sires, Brahman sires can be expected to increase gestation length, increase birth weight, reduce the percentage of unassisted calvings, and increase weaning weight. In only 1 study (Browning et al., 1995) was weaning weight lower for Brahman-sired progeny. Results of the latter study probably are a reflection of the fact that the Brahman-sired progeny were straightbred, whereas the Angus-sired progeny were 2-breed crosses. Thus, direct genetic and direct heterotic effects are partially confounded in this comparison. Increased dystocia associated with the use of Brahman bulls has been addressed previously (Thrift et al., 2002).

Brahman Versus Other Bos indicus Sire Breeds. Compared with Brahman sires, Sahiwal sires contribute to a longer gestation length, lighter birth weights (resulting in a higher percentage of unassisted calvings), and lighter weaning weights. In the Texas study by Paschal et 
al. (1991), no difference was evident between Gray- and Red Brahmansired progeny; however, relative to Gray Brahman, Indu-Brazil-sired progeny were heavier at birth, but not at weaning. Gir-sired progeny were lighter at birth and weaning than Gray Brahman-sired progeny. Nelloreand Gray Brahman-sired progeny performed similarly, with some indication that Nellore sires contributed to a slightly longer gestation length. In 2 studies (Herring et al., 1996; Cundiff, 2005), Brahman-sired progeny were heavier at birth and weaning than Boran-sired progeny.

Brahman Versus Bos indicusDerivative Sire Breeds. Cundiff (2005) indicated that in the more temperate environment of Nebraska, Brahman sires contributed to a longer gestation length and higher birth weight (resulting in a lower percentage of unassisted calvings) but similar weaning weights compared with Brangus and Beefmaster sires. Birth and weaning weights were similar for Brahman- and Beefmaster-sired progeny in Arkansas (Brown et al., 1997).

\section{Brahman Versus non-Bos} indicus Subtropically Adapted Sire Breeds. Relative to Brahman sires, Tuli, Romosinuano, Bonsmara, and Senepol sires result in a shorter gestation length and substantially lighter birth and weaning weights. Because of the lighter birth weights, percentage of unassisted calvings consistently favors these subtropically adapted sire breeds over the Brahman. In the study by Browning et al. (1995), weaning weights were similar for Brahman- and Tuli-sired progeny. As indicated previously, these results are probably because the Brahmansired progeny were straightbreds and the Tuli-sired progeny were 2-breed crosses.

Bos indicus-Derivative Versus Traditional Bos taurus Sire Breeds. In general, Brangus-, Beefmaster-, and Santa Gertrudis-sired progeny are consistently heavier at birth and weaning than Angus-, Hereford-, and Red Poll-sired progeny. In the study by Wheeler et al. (2006), Brangus and Beefmaster sires contributed to slightly longer gestation lengths and higher birth weights but percentage of unassisted calvings was similar to those for Angus and Hereford sires. In 1 study (Oxford et al., 2006), Santa Gertrudis-sired progeny were heavier at birth and weaning than Angus-, Hereford-, and Red Poll-sired progeny. Birth and weaning weights were similar for Santa Gertrudis- and Charolais-sired progeny.

Non-Bos indicus Subtropically Adapted Versus Traditional Bos taurus Sire Breeds. Relative to Angus and Hereford sires, Tuli, Romosinuano, Bonsmara, and Senepol sires increase the gestation length but do not consistently increase the birth weight. In the 3 cases (Cundiff, 2005; Wheeler et al., 2006; Thrift et al., 1986, Kentucky study) in which birth weights were greater for progeny of these non- $B$. indicus subtropical sire breeds, the increase averaged less than $1.5 \mathrm{~kg}$. With the exception of the comparison involving Romosinuano and Hereford sires (Wheeler et al., 2006), in which Romosinuanosired progeny experienced less dystocia, percentage of unassisted calvings was similar for the non- $B$. indicus subtropically adapted sire breeds and the $B$. taurus sire breeds. In 1 study (Brown and Lalman, 2008), Charolais-sired progeny were significantly heavier at birth than Romosinuano- and Bonsmara-sired progeny. In 11 studies, weaning weights were similar; however, in 16 studies, weaning weights were significantly lighter for the non- $B$. indicus subtropically adapted sire breeds relative to the $B$. taurus sire breeds.

\section{Postweaning Traits}

Postweaning trait (postweaning ADG, feedlot ADG, final feedlot BW) comparisons involving progeny sired by subtropically adapted beef sire breeds are summarized in Table 2. Results are summarized by comparing sire breed means for each trait.

Brahman Versus Traditional Bos taurus Sire Breeds. Results comparing postweaning ADG for Brahman- and Angus-sired progeny are inconsistent. Paschal et al. (1991) indicated that compared with Angussired progeny, Gray Brahman-sired progeny had a higher postweaning ADG; however, Brown et al. (1993a) indicated a lower postweaning ADG for Brahman-sired progeny. Most studies indicated similar feedlot ADG and final feedlot BW for Brahman- and B. taurus-sired progeny; however, Cundiff (2005) indicated lower feedlot ADG and final feedlot BW for Brahman-sired progeny. These latter results are as expected because cattle involved in the study by Cundiff (2005) were weaned in the fall and fed during the winter season at the US Meat Animal Research Center (Clay Center, NE). It has been established that Brahman-sired cattle have lower feedlot performance when fed at temperate locations during the winter season (Thrift and Thrift, 2005). In contrast to the results of Cundiff (2005), Paschal et al. (1991) indicated a postweaning advantage for Gray Brahman-sired over Angus-sired progeny fed during the winter season at a central Texas location.

\section{Brahman Versus Other Bos} indicus Sire Breeds Sahiwal-, Gir-, and Boran-sired progeny have lower postweaning performance than Brahman-sired progeny. Final feedlot BW appears to be reduced by slightly less than $40 \mathrm{~kg}$. When evaluated under central Texas conditions, Red Brahman-, Indu-Brazil-, and Nelloresired progeny performed similarly to Gray Brahman-sired progeny during the postweaning period (Paschal et al., 1995).

Brahman Versus Bos indicusDerivative Sire Breeds. Cundiff (2005) indicated that Brangus- and Beefmaster-sired progeny expressed greater feedlot ADG and final feedlot BW than Brahman-sired progeny. As indicated previously, these results are likely associated with the lower feedlot performance of Brahman-sired progeny when fed during the winter season at a temperate location (Thrift and Thrift, 2005).

Brahman Versus non-Bos indicus Subtropically Adapted Sire Breeds. Brahman-sired progeny 
Table 2. Postweaning trait comparisons involving progeny sired by subtropically adapted beef sire breeds

\begin{tabular}{|c|c|c|c|c|c|c|}
\hline \multirow[b]{3}{*}{ Sire breed comparison } & \multicolumn{6}{|c|}{ Trait $^{1}$} \\
\hline & \multicolumn{2}{|c|}{ Postweaning } & \multicolumn{4}{|c|}{ Feedlot } \\
\hline & $\mathbf{N}$ & ADG, kg & $\mathbf{N}$ & ADG, $\mathbf{k g}$ & $\mathbf{N}$ & FBW, kg \\
\hline \multicolumn{7}{|c|}{ Brahman vs. traditional $B$. taurus sire breeds } \\
\hline \multicolumn{7}{|c|}{ Koch et al. (1982) } \\
\hline Brahman & & & & & 128 & 477.7 \\
\hline Angus/Hereford & & & & & 140 & 466.5 \\
\hline Difference & & & & & & $11.2 \mathrm{NS}$ \\
\hline \multicolumn{7}{|c|}{ Sanders and Paschal $(1987)^{2}$} \\
\hline Zebu & & & 79 & 1.49 & 79 & 489.5 \\
\hline Angus & & & 28 & 1.58 & 28 & 480.8 \\
\hline Difference & & & & $-0.09 \mathrm{NT}$ & & $8.7 \mathrm{NT}$ \\
\hline \multicolumn{7}{|l|}{ Paschal et al. (1995) } \\
\hline Gray Brahman & 42 & 0.39 & 20 & 1.60 & 20 & 499.7 \\
\hline Angus & 33 & 0.22 & 18 & 1.57 & 18 & 468.4 \\
\hline Difference & & $0.17^{*}$ & & $0.03 \mathrm{NS}$ & & $31.3^{*}$ \\
\hline \multicolumn{7}{|l|}{ Brown et al. (1993a) } \\
\hline Brahman & 180 & 0.32 & & & & \\
\hline Angus & 251 & 0.37 & & & & \\
\hline Difference & & $-0.05^{*}$ & & & & \\
\hline \multicolumn{7}{|l|}{ Baker et al. (2001b) } \\
\hline Brahman & & & & & & 573.8 \\
\hline Angus & & & & & & 576.3 \\
\hline Difference & & & & & & $-2.5 \mathrm{NS}$ \\
\hline \multicolumn{7}{|l|}{ Cundiff (2005) } \\
\hline Brahman & & & 119 & 1.20 & 119 & 544.8 \\
\hline Angus & & & 467 & 1.42 & 467 & 594.7 \\
\hline Difference & & & & $-0.22^{*}$ & & $-49.9^{*}$ \\
\hline \multicolumn{7}{|l|}{ Amen et al. (2007b) } \\
\hline Brahman & & & & & 133 & 508.2 \\
\hline Angus & & & & & 128 & 524.4 \\
\hline Difference & & & & & & $-16.2 \mathrm{NS}$ \\
\hline \multicolumn{7}{|l|}{ Baker et al. (2001b) } \\
\hline Brahman & & & & & & 573.8 \\
\hline Hereford & & & & & & 562.5 \\
\hline Difference & & & & & & $11.3 \mathrm{NS}$ \\
\hline \multicolumn{7}{|l|}{ Cundiff (2005) } \\
\hline Brahman & & & 119 & 1.20 & 119 & 544.8 \\
\hline Hereford & & & 448 & 1.38 & 448 & 580.2 \\
\hline Difference & & & & $-0.18^{*}$ & & $-35.4^{*}$ \\
\hline \multicolumn{7}{|c|}{ Brahman vs. other $B$. indicus sire breeds } \\
\hline \multicolumn{7}{|c|}{ Koch et al. (1982) } \\
\hline Brahman & & & & & 128 & 477.7 \\
\hline Sahiwal & & & & & 141 & 445.4 \\
\hline Difference & & & & & & $32.3^{*}$ \\
\hline \multicolumn{7}{|l|}{ Crouse et al. (1989) } \\
\hline Brahman & & & & & 84 & 460.0 \\
\hline Sahiwal & & & & & 88 & 418.0 \\
\hline \multirow{2}{*}{\multicolumn{7}{|c|}{ Paschal et al. (1995) }} \\
\hline & & & & & & \\
\hline Gray Brahman & 42 & 0.39 & 20 & 1.60 & 20 & 499.7 \\
\hline Red Brahman & 43 & 0.39 & 21 & 1.57 & 21 & 511.0 \\
\hline Difference & & $0.00 \mathrm{NS}$ & & $0.03 \mathrm{NS}$ & & $-11.3 \mathrm{NS}$ \\
\hline Paschal et al. (1995) & & & & & & \\
\hline Gray Brahman & 42 & 0.39 & 20 & 1.60 & 20 & $\begin{array}{l}499.7 \\
\quad \text { Continued }\end{array}$ \\
\hline
\end{tabular}


Table 2 (Continued). Postweaning trait comparisons involving progeny sired by subtropically adapted beef sire breeds

\begin{tabular}{|c|c|c|c|c|c|c|}
\hline \multirow[b]{3}{*}{ Sire breed comparison } & \multicolumn{6}{|c|}{ Trait $^{1}$} \\
\hline & \multicolumn{2}{|c|}{ Postweaning } & \multicolumn{4}{|c|}{ Feedlot } \\
\hline & $\mathbf{N}$ & ADG, kg & $\mathbf{N}$ & ADG, kg & $\mathbf{N}$ & FBW, $\mathbf{k g}$ \\
\hline Gir & 42 & 0.37 & 27 & 1.47 & 27 & 471.9 \\
\hline Difference & & $0.02 \mathrm{NS}$ & & $0.13 \mathrm{NS}$ & & $27.8^{*}$ \\
\hline \multicolumn{7}{|l|}{ Paschal et al. (1995) } \\
\hline Gray Brahman & 42 & 0.39 & 20 & 1.60 & 20 & 499.7 \\
\hline Indu-Brazil & 39 & 0.35 & 19 & 1.60 & 19 & 491.7 \\
\hline Difference & & $0.04^{*}$ & & $0.00 \mathrm{NS}$ & & $8.0 \mathrm{NS}$ \\
\hline \multicolumn{7}{|l|}{ Paschal et al. (1995) } \\
\hline Gray Brahman & 42 & 0.39 & 20 & 1.60 & 20 & 499.7 \\
\hline Nellore & 46 & 0.36 & 21 & 1.50 & 21 & 480.1 \\
\hline Difference & & $0.03 \mathrm{NS}$ & & $0.10 \mathrm{NS}$ & & 19.6 NS \\
\hline \multicolumn{7}{|l|}{ Cundiff (2005) } \\
\hline Brahman & & & 119 & 1.20 & 119 & 544.8 \\
\hline Nellore & & & 101 & 1.26 & 101 & 557.5 \\
\hline Difference & & & & $-0.06 \mathrm{NS}$ & & $-12.7 \mathrm{NS}$ \\
\hline \multicolumn{7}{|l|}{ Herring et al. (1996) } \\
\hline Brahman & & & 40 & 1.33 & 40 & 504.9 \\
\hline Boran & & & 59 & 1.22 & 59 & 446.8 \\
\hline Difference & & & & $0.11^{*}$ & & $58.1^{*}$ \\
\hline \multicolumn{7}{|l|}{ Cundiff (2005) } \\
\hline Brahman & & & 119 & 1.20 & 119 & 544.8 \\
\hline Boran & & & 151 & 1.13 & 151 & 510.8 \\
\hline Difference & & & & $0.07^{*}$ & & $34.0^{*}$ \\
\hline \multicolumn{7}{|c|}{ Brahman vs. B. indicus-derivative sire breeds } \\
\hline \multicolumn{7}{|c|}{ Cundiff (2005) } \\
\hline Brahman & & & 119 & 1.20 & 119 & 544.8 \\
\hline Brangus & & & 107 & 1.36 & 107 & 584.7 \\
\hline Difference & & & & $-0.16^{*}$ & & $-39.9^{*}$ \\
\hline \multicolumn{7}{|l|}{ Cundiff (2005) } \\
\hline Brahman & & & 119 & 1.20 & 119 & 544.8 \\
\hline Beefmaster & & & 103 & 1.41 & 103 & 602.4 \\
\hline Difference & & & & $-0.21^{*}$ & & $-57.6^{*}$ \\
\hline \multicolumn{7}{|c|}{ Brahman vs. non- $B$. indicus subtropically adapted sire breeds } \\
\hline \multicolumn{7}{|c|}{ 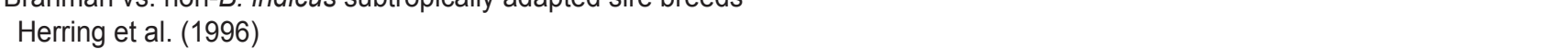 } \\
\hline Brahman & & & 40 & 1.33 & 40 & 504.9 \\
\hline Tuli & & & 43 & 1.18 & 43 & 454.3 \\
\hline Difference & & & & $0.15^{*}$ & & $50.6^{*}$ \\
\hline \multicolumn{7}{|l|}{ Baker et al. (2001b) } \\
\hline Brahman & & & & & & 573.8 \\
\hline Tuli & & & & & & 525.6 \\
\hline Difference & & & & & & $48.2^{*}$ \\
\hline \multicolumn{7}{|l|}{ Cundiff (2005) } \\
\hline Brahman & & & 119 & 1.20 & 119 & 544.8 \\
\hline Tuli & & & 162 & 1.15 & 162 & 508.0 \\
\hline Difference & & & & $0.05^{\star}$ & & $36.8^{*}$ \\
\hline \multicolumn{7}{|l|}{ Cundiff (2005) } \\
\hline Brahman & & & 119 & 1.20 & 119 & 544.8 \\
\hline Romosinuano & & & 102 & 1.23 & 102 & 535.2 \\
\hline Difference & & & & -0.03 NS & & 9.6 NS \\
\hline \multicolumn{7}{|l|}{ Cundiff (2005) } \\
\hline Brahman & & & 119 & 1.20 & 119 & 544.8 \\
\hline
\end{tabular}


Table 2 (Continued). Postweaning trait comparisons involving progeny sired by subtropically adapted beef sire breeds

\begin{tabular}{|c|c|c|c|c|c|c|}
\hline \multirow[b]{3}{*}{ Sire breed comparison } & \multicolumn{6}{|c|}{ Trait $^{1}$} \\
\hline & \multicolumn{2}{|c|}{ Postweaning } & \multicolumn{4}{|c|}{ Feedlot } \\
\hline & $\mathbf{N}$ & ADG, $\mathrm{kg}$ & $\mathbf{N}$ & ADG, $\mathbf{k g}$ & $\mathbf{N}$ & FBW, $\mathrm{kg}$ \\
\hline $\begin{array}{l}\text { Bonsmara } \\
\text { Difference }\end{array}$ & & & 104 & $\begin{array}{c}1.28 \\
-0.08^{*}\end{array}$ & 104 & $\begin{array}{l}552.5 \\
-7.7 \mathrm{NS}\end{array}$ \\
\hline \multicolumn{7}{|c|}{ Sanders and Paschal $(1987)^{2}$} \\
\hline Zebu & & & 79 & 1.49 & 79 & 489.5 \\
\hline Senepol & & & 14 & 1.61 & 14 & 490.3 \\
\hline Difference & & & & $-0.12 \mathrm{NT}$ & & $-0.8 \mathrm{NT}$ \\
\hline
\end{tabular}

$B$. indicus-derivative vs. traditional $B$. taurus sire breeds

DeRouen et al. (2000), Louisiana feedlot

Brangus

Angus

Difference

DeRouen et al. (2000), Oklahoma feedlot

Brangus

Angus

Difference

Wheeler et al. (2006)

Brangus

Angus

Difference

Wheeler et al. (2006)

Brangus

Hereford

Difference

DeRouen et al. (2000), Louisiana feedlot

Brangus

Gelbvieh

Difference

DeRouen et al. (2000), Oklahoma feedlot

Brangus

Gelbvieh

Difference

DeRouen et al. (2000), Louisiana feedlot

Gelbray

Angus

Difference

DeRouen et al. (2000), Oklahoma feedlot

Gelbray

Angus

Difference

DeRouen et al. (2000), Louisiana feedlot

Gelbray

Gelbvieh

Difference

DeRouen et al. (2000), Oklahoma feedlot

Gelbray

Gelbvieh

Difference

$\begin{array}{llll}27 & 1.17 & 27 & 551.0 \\ 30 & 1.17 & 30 & 577.0 \\ & 0.00 \mathrm{NS} & & -26.0 \mathrm{NS}\end{array}$

$39 \quad 1.68$

$39 \quad 557.0$

$30 \quad 1.62$

0.06 NS

$30 \quad 562.0$

$107 \quad 1.36$

$103 \quad 1.43$

$107 \quad 569.7$

$-0.07^{*}$

$103 \quad 582.0$

$107 \quad 1.36$

$102 \quad 1.37$

$-0.01 \mathrm{NS}$

$107 \quad 569.7$

$102 \quad 564.7$

$5.0 \mathrm{NS}$

$27 \quad 1.17$

$27 \quad 551.0$

26

1.12

0.05 NS

$26 \quad 603.0$

$39 \quad 1.68$

$23 \quad 1.55$

0.13 NS

$39 \quad 557.0$

$23 \quad 554.0$

$3.0 \mathrm{NS}$

$31 \quad 0.86$

$31 \quad 527.0$

$30 \quad 1.17$

$30 \quad 577.0$

$-0.31^{*}$

$-50.0^{*}$

$25 \quad 1.61$

$25 \quad 549.0$

$30 \quad 1.62$

$-0.01 \mathrm{NS}$

$30 \quad 562.0$

$-13.0 \mathrm{NS}$

$31 \quad 0.86$

$31 \quad 527.0$

$26 \quad 1.12$

$-0.26^{*}$

$26 \quad 603.0$

$$
1.61
$$

25

1.61

1.55

0.06 NS

$25 \quad 549.0$

$23 \quad 544.0$

Wheeler et al. (2006)

Beefmaster

Angus

Difference

1.43

-0.02 NS

$103 \quad 587.9$

$103 \quad 582.0$ 
Table 2 (Continued). Postweaning trait comparisons involving progeny sired by subtropically adapted beef sire breeds

\begin{tabular}{|c|c|c|c|c|c|c|}
\hline \multirow[b]{3}{*}{ Sire breed comparison } & \multicolumn{6}{|c|}{ Trait $^{1}$} \\
\hline & \multicolumn{2}{|c|}{ Postweaning } & \multicolumn{4}{|c|}{ Feedlot } \\
\hline & $\mathbf{N}$ & ADG, kg & $\mathbf{N}$ & ADG, kg & $\mathbf{N}$ & FBW, kg \\
\hline \multicolumn{7}{|l|}{ Wheeler et al. (2006) } \\
\hline Beefmaster & & & 103 & 1.41 & 103 & 587.9 \\
\hline Hereford & & & 102 & 1.37 & 102 & 564.7 \\
\hline Difference & & & & $0.04^{*}$ & & $23.2^{*}$ \\
\hline
\end{tabular}

$B$. indicus-derivative vs. non- $B$. indicus subtropically adapted sire breeds

Wheeler et al. (2006)

Brangus

Romosinuano

Difference

Wheeler et al. (2006)

Brangus

Bonsmara

Difference

Wheeler et al. (2006)

Beefmaster

Romosinuano

Difference

Wheeler et al. (2006)

Beefmaster

Bonsmara

Difference

$\begin{array}{lllc}107 & 1.36 & 107 & 569.7 \\ 102 & 1.23 & 102 & 521.6 \\ & 0.13^{*} & & 48.1^{*} \\ & & & \\ 107 & 1.36 & 107 & 569.7 \\ 104 & 1.27 & 104 & 537.5 \\ & 0.09^{*} & & 32.2^{*} \\ & & & \\ 103 & 1.41 & 103 & 587.9 \\ 102 & 1.23 & 102 & 521.6 \\ & 0.18^{*} & & 66.3^{*} \\ 103 & 1.41 & 103 & 587.9 \\ 104 & 1.27 & 104 & 537.5 \\ & 0.14^{*} & & 50.4^{*}\end{array}$

Non- $B$. indicus subtropically adapted vs. traditional $B$. taurus sire breeds

Baker et al. (2001b)

Tuli

Angus

Difference

Cundiff (2005)

Tuli

Angus

Difference

Baker et al. (2001b)

Tuli

Hereford

Difference

Cundiff (2005)

Tuli

Hereford

Difference

Cundiff (2005)

Bonsmara

Angus

Difference

Cundiff (2005)

Bonsmara

Hereford

Difference

Sanders and Paschal (1987)

Senepol

525.6

576.3

$-50.7^{*}$

162

142

1.15

1.42

$-0.27^{*}$

$162 \quad 508.0$

$467 \quad 594.7$

$-86.7^{*}$

525.6

562.5

$-36.9^{*}$

1.15

162

$448 \quad 1.38$

$-0.23^{*}$

$448 \quad 580.2$

$-72.2^{*}$

$104 \quad 1.27$

$104 \quad 552.5$

467

1.42

$-0.15^{*}$

$467 \quad 594.7$

$104 \quad 1.27$

$448 \quad 1.38$

$-0.11^{*}$

$104 \quad 552.5$

$448 \quad 580.2$

$14 \quad 1.61$

$-27.7^{*}$

$14 \quad 490.3$

Continued 
Table 2 (Continued). Postweaning trait comparisons involving progeny sired by subtropically adapted beef sire breeds

\begin{tabular}{|c|c|c|c|c|c|c|}
\hline \multirow[b]{3}{*}{ Sire breed comparison } & \multicolumn{6}{|c|}{ Trait $^{1}$} \\
\hline & \multicolumn{2}{|c|}{ Postweaning } & \multicolumn{4}{|c|}{ Feedlot } \\
\hline & $\mathbf{N}$ & ADG, kg & $\mathbf{N}$ & ADG, kg & $\mathbf{N}$ & FBW, kg \\
\hline Angus & & & 28 & 1.58 & 28 & 480.8 \\
\hline Difference & & & & $0.03 \mathrm{NT}$ & & $9.5 \mathrm{NT}$ \\
\hline \multicolumn{7}{|c|}{ Chase et al. (1998), phase II } \\
\hline Senepol & & & 56 & 1.21 & 56 & 414.0 \\
\hline Hereford & & & 62 & 1.37 & 62 & 414.5 \\
\hline Difference & & & & $-0.16^{* *}$ & & $-0.5 \mathrm{NS}$ \\
\hline \multicolumn{7}{|l|}{ Cundiff (2005) } \\
\hline Romosinuano & & & 102 & 1.23 & 102 & 535.2 \\
\hline Angus & & & 467 & 1.42 & 467 & 594.7 \\
\hline Difference & & & & $-0.19^{*}$ & & $-59.5^{\star}$ \\
\hline \multicolumn{7}{|l|}{ Phillips et al. (2006) } \\
\hline Romosinuano & 99 & 0.57 & 99 & 1.27 & 99 & 494.0 \\
\hline Angus & 51 & 0.69 & 51 & 1.38 & 51 & 510.0 \\
\hline Difference & & $-0.12^{* *}$ & & $-0.11 \mathrm{NS}$ & & $-16.0 \mathrm{NS}$ \\
\hline \multicolumn{7}{|l|}{ Cundiff (2005) } \\
\hline Romosinuano & & & 102 & 1.23 & 102 & 535.2 \\
\hline Hereford & & & 448 & 1.38 & 448 & 580.2 \\
\hline Difference & & & & $-0.15^{\star}$ & & $-45.0^{*}$ \\
\hline \multicolumn{7}{|c|}{ Brown et al. (2008), heifers managed in drylot postweaning } \\
\hline Romosinuano & 12 & 0.84 & & & & \\
\hline Hereford & 12 & 1.04 & & & & \\
\hline Difference & & $-0.20 \dagger$ & & & & \\
\hline \multicolumn{7}{|c|}{ Brown et al. (2008), heifers managed in drylot postweaning } \\
\hline Romosinuano & 12 & 0.84 & & & & \\
\hline Gelbvieh & 15 & 1.14 & & & & \\
\hline Difference & & $-0.30 \dagger$ & & & & \\
\hline \multicolumn{7}{|c|}{ Brown et al. (2008), heifers managed in drylot postweaning } \\
\hline Romosinuano & 12 & 0.84 & & & & \\
\hline Charolais & 14 & 1.07 & & & & \\
\hline Difference & & $-0.23 \dagger$ & & & & \\
\hline \multicolumn{7}{|c|}{ Brown et al. (2008), heifers managed in drylot postweaning } \\
\hline Bonsmara & 15 & 0.99 & & & & \\
\hline Hereford & 12 & 1.04 & & & & \\
\hline Difference & & $-0.05 \mathrm{NS}$ & & & & \\
\hline \multicolumn{7}{|c|}{ Brown et al. (2008), heifers managed in drylot postweaning } \\
\hline Bonsmara & 15 & 0.99 & & & & \\
\hline Gelbvieh & 15 & 1.14 & & & & \\
\hline Difference & & $-0.15 \dagger$ & & & & \\
\hline \multicolumn{7}{|c|}{ Brown et al. (2008), heifers managed in drylot postweaning } \\
\hline Bonsmara & 15 & 0.99 & & & & \\
\hline Charolais & 14 & 1.07 & & & & \\
\hline Difference & & $-0.08 \mathrm{NS}$ & & & & \\
\hline \multicolumn{7}{|c|}{$\begin{array}{l}\text { Brown et al. (2008), heifers managed on wheat pasture } \\
\text { postweaning }\end{array}$} \\
\hline Romosinuano & 15 & 0.48 & & & & \\
\hline Hereford & 15 & 0.62 & & & & \\
\hline Difference & & $-0.14 \dagger$ & & & & \\
\hline \multicolumn{7}{|c|}{$\begin{array}{l}\text { Brown et al. (2008), heifers managed on wheat pasture } \\
\text { postweaning }\end{array}$} \\
\hline Romosinuano & 15 & 0.48 & & & & \\
\hline Gelbvieh & 18 & 0.61 & & & & \\
\hline Difference & & $-0.13 \dagger$ & & & & \\
\hline
\end{tabular}


Table 2 (Continued). Postweaning trait comparisons involving progeny sired by subtropically adapted beef sire breeds

\begin{tabular}{|c|c|c|c|c|c|c|}
\hline \multirow[b]{3}{*}{ Sire breed comparison } & \multicolumn{6}{|c|}{ Trait $^{1}$} \\
\hline & \multicolumn{2}{|c|}{ Postweaning } & \multicolumn{4}{|c|}{ Feedlot } \\
\hline & $\mathbf{N}$ & ADG, $\mathbf{k g}$ & $\mathbf{N}$ & ADG, kg & $\mathbf{N}$ & FBW, kg \\
\hline
\end{tabular}

Brown et al. (2008), heifers managed on wheat pasture postweaning

Romosinuano

Charolais

Difference

$12 \quad 0.48$

$18 \quad 0.60$

Brown et al. (2008), heifers managed on wheat pasture

$-0.12 \dagger$

postweaning

Bonsmara

Hereford

Difference

$17 \quad 0.50$

$12 \quad 1.04$

Brown et al. (2008), heifers managed on wheat pasture $-0.54 \dagger$

postweaning

Bonsmara

Gelbvieh

Difference

0.50

$18 \quad 0.61$

Brown et al. (2008), heifers managed on wheat pasture postweaning

Bonsmara

Charolais

Difference

Brown et al. (2008), steers managed in drylot postweaning Romosinuano

Hereford

Difference

Brown et al. (2008), steers managed in drylot postweaning Romosinuano

Gelbvieh

Difference

Brown et al. (2008), steers managed in drylot postweaning

Romosinuano

Charolais

Difference

Brown et al. (2008), steers managed in drylot postweaning

Bonsmara

Hereford

Difference

Brown et al. (2008), steers managed in drylot postweaning

Bonsmara

Gelbvieh

Difference

Brown et al. (2008), steers managed in drylot postweaning

Bonsmara

Charolais

Difference

$-0.11 \dagger$

$15 \quad 0.50$

$14 \quad 0.60$

$-0.10 \dagger$

$13 \quad 1.02$

$14 \quad 1.02$

$0.00 \mathrm{NS}$

$13 \quad 1.02$

$13 \quad 1.03$

-0.01 NS

$13 \quad 1.02$

$12 \quad 1.15$

$-0.13 \dagger$

$18 \quad 1.05$

$14 \quad 1.02$

0.03 NS

$18 \quad 1.05$

$13 \quad 1.03$

0.02 NS

$18 \quad 1.05$

$12 \quad 1.15$

$-0.10 \dagger$

Brown et al. (2008), steers managed on wheat pasture postweaning

Romosinuano

Hereford

Difference

Brown et al. (2008), steers managed on wheat pasture

postweaning

Romosinuano 
Table 2 (Continued). Postweaning trait comparisons involving progeny sired by subtropically adapted beef sire breeds

\begin{tabular}{|c|c|c|c|c|c|c|}
\hline \multirow[b]{3}{*}{ Sire breed comparison } & \multicolumn{6}{|c|}{ Trait $^{1}$} \\
\hline & \multicolumn{2}{|c|}{ Postweaning } & \multicolumn{4}{|c|}{ Feedlot } \\
\hline & $\mathbf{N}$ & ADG, kg & $\mathbf{N}$ & ADG, kg & $\mathbf{N}$ & FBW, kg \\
\hline Gelbvieh & 16 & 0.71 & & & & \\
\hline Difference & & $-0.08 \mathrm{NS}$ & & & & \\
\hline \multicolumn{7}{|c|}{$\begin{array}{l}\text { Brown et al. (2008), steers managed on wheat pasture } \\
\text { postweaning }\end{array}$} \\
\hline Romosinuano & 15 & 0.63 & & & & \\
\hline Charolais & 15 & 0.72 & & & & \\
\hline Difference & & $-0.09 \dagger$ & & & & \\
\hline \multicolumn{7}{|c|}{$\begin{array}{l}\text { Brown et al. (2008), steers managed on wheat pasture } \\
\text { postweaning }\end{array}$} \\
\hline Bonsmara & 30 & 0.68 & & & & \\
\hline Hereford & 23 & 0.74 & & & & \\
\hline Difference & & $-0.06 \mathrm{NS}$ & & & & \\
\hline \multicolumn{7}{|c|}{$\begin{array}{l}\text { Brown et al. (2008), steers managed on wheat pasture } \\
\text { postweaning }\end{array}$} \\
\hline Bonsmara & 30 & 0.68 & & & & \\
\hline Gelbvieh & 16 & 0.71 & & & & \\
\hline Difference & & $-0.03 \mathrm{NS}$ & & & & \\
\hline \multicolumn{7}{|c|}{$\begin{array}{l}\text { Brown et al. (2008), steers managed on wheat pasture } \\
\text { postweaning }\end{array}$} \\
\hline Bonsmara & 30 & 0.68 & & & & \\
\hline Charolais & 15 & 0.72 & & & & \\
\hline Difference & & $-0.04 \mathrm{NS}$ & & & & \\
\hline
\end{tabular}

${ }^{1} \mathrm{FBW}=$ final feedlot BW.

2Zebu collectively refers to average for Gray Brahman, Red Brahman, Gir, Indu-Brazil, and Nellore.

${ }^{*} P<0.05 ; \dagger P<0.10 ; \mathrm{NS}=$ not significant $(P>0.10)$; NT = not tested.

consistently outperform Tuli-sired progeny during the postweaning period. The final feedlot BW advantage for Brahman-sired progeny averaged approximately 45 kg. Romosinuano-, Bonsmara-, and Senepol-sired progeny performed similarly to Brahman-sired progeny.

Bos indicus-Derivative Versus Traditional Bos taurus Sire Breeds. In general, Brangus- and Beefmaster-sired progeny perform similarly to or slightly below Angus-, Hereford-, and Gelbvieh-sired progeny during the postweaning period. In contrast, Wheeler et al. (2006) indicated superior postweaning performance for Beefmaster-sired over Hereford-sired, but not Angus-sired, progeny. The results of DeRouen et al. (2000) were dependent on feedlot location, but in general indi- cated equal or subpar performance of Gelbray-sired progeny compared with Angus- or Gelbvieh-sired progeny.

Bos indicus-Derivative Versus non-Bos indicus Subtropically Adapted Sire Breeds. Wheeler et al. (2006) indicated superior postweaning performance for Brangus- and Beefmaster-sired progeny relative to Romosinuano- and Bonsmara-sired progeny. Specifically, advantages for final feedlot BW were substantial for the Brangus- and Beefmaster-sired progeny, averaging approximately 50 $\mathrm{kg}$.

Non-Bos indicus Subtropically Adapted Versus Traditional Bos taurus Sire Breeds. Feedlot ADG and final feedlot BW of Tuli-, Bonsmara-, and Romosinuano-sired progeny are consistently lower than those of Angus- and Hereford-sired progeny. The results of Phillips et al. (2006), and the extensive results of Brown et al. (2008), indicate a consistent postweaning ADG advantage for Angus-, Hereford-, Gelbvieh-, and Charolais-sired progeny relative to Romosinuano- and Bonsmara-sired progeny.

\section{Carcass Traits}

Carcass trait comparisons (hot carcass weight, YG, QG, marbling score, Warner-Bratzler shear force value, LM area) involving progeny sired by subtropically adapted beef sire breeds are summarized in Table 3. Results are summarized by comparing sire breed means for each trait.

Brahman Versus Traditional Bos taurus Sire Breeds. Relative to Brahman-sired progeny, Angus- 
Table 3. Carcass trait comparisons involving progeny sired by subtropically adapted beef sire breeds

\begin{tabular}{|c|c|c|c|c|c|c|c|}
\hline \multirow[b]{2}{*}{ Sire breed comparison } & \multicolumn{7}{|c|}{ Trait $^{1}$} \\
\hline & $\mathbf{N}$ & HCW, kg & YG & QG & MBS & WBS, kg L & LM area, $\mathrm{cm}^{2}$ \\
\hline \multicolumn{8}{|c|}{ Brahman vs. traditional $B$. taurus sire breeds } \\
\hline \multicolumn{8}{|c|}{ Koch et al. (1982) } \\
\hline Brahman & 128 & 308.2 & 3.7 & 8.8 & 9.4 & 3.9 & 70.5 \\
\hline Angus/Hereford & 140 & 296.4 & 3.8 & 10.1 & 11.4 & 3.4 & 69.1 \\
\hline Difference & & $11.8 \mathrm{NS}$ & $-0.1 \mathrm{NS}$ & $-1.3^{*}$ & $-2.0^{*}$ & $0.5^{*}$ & 1.4 NS \\
\hline \multicolumn{8}{|c|}{ Sanders and Paschal $(1987)^{2,3}$} \\
\hline Zebu & 79 & 297.0 & 2.6 & $\mathrm{Gd}$ & SI & & 75.9 \\
\hline Angus & 28 & 285.4 & 2.6 & $\mathrm{Ch}$ & $\mathrm{Sm}$ & & 75.8 \\
\hline Difference & & 11.6 NT & $0.0 \mathrm{NT}$ & - NT & - NT & & $0.1 \mathrm{NT}$ \\
\hline \multicolumn{8}{|l|}{ Paschal et al. (1995) } \\
\hline Gray Brahman & 19 & 300.1 & 2.8 & 344.2 & 347.7 & & 75.5 \\
\hline Angus & 18 & 275.8 & 2.5 & 391.2 & 410.2 & & 76.6 \\
\hline Difference & & $24.3^{*}$ & $0.3^{*}$ & $-47.0^{*}$ & $-62.5^{*}$ & & $-1.1 \mathrm{NS}$ \\
\hline \multicolumn{8}{|l|}{ DeRouen et al. $(1992)^{4}$} \\
\hline Brahman & 216 & 230.0 & & & 3.2 & 13.2 & 62.0 \\
\hline Angus & 302 & 243.0 & & & 4.7 & 9.5 & 66.0 \\
\hline Difference & & $-13.0^{*}$ & & & $-1.5^{*}$ & $3.7^{*}$ & $-4.0^{*}$ \\
\hline \multicolumn{8}{|l|}{ Brown et al. (1999) } \\
\hline Brahman & 150 & 333.3 & 2.7 & & 3.6 & & 84.0 \\
\hline Angus & 201 & 317.8 & 2.8 & & 3.8 & & 82.7 \\
\hline Difference & & $15.5 \mathrm{NS}$ & $-0.1 \mathrm{NS}$ & & $-0.2 \mathrm{NS}$ & & $1.3 \mathrm{NS}$ \\
\hline \multicolumn{8}{|l|}{ Baker et al. (2001b) } \\
\hline Brahman & & 334.1 & 3.1 & & 234.5 & 5.3 & 79.1 \\
\hline Angus & & 332.7 & 3.4 & & 269.4 & 4.0 & 78.0 \\
\hline Difference & & $1.4 \mathrm{NS}$ & $-0.3^{*}$ & & $-34.9^{*}$ & $1.3^{*}$ & $1.1 \mathrm{NS}$ \\
\hline \multicolumn{8}{|l|}{ Wheeler et al. $(2001)^{5}$} \\
\hline Brahman & 119 & 332.0 & 3.3 & 30.0 & 472.9 & 6.0 & 72.9 \\
\hline Angus & 127 & 352.0 & 3.5 & 84.0 & 553.0 & 4.1 & 75.8 \\
\hline Difference & & $-20.0^{*}$ & $-0.2 \mathrm{NS}$ & $-54.0^{*}$ & $-80.1^{*}$ & $1.9^{*}$ & $-2.9^{*}$ \\
\hline \multicolumn{8}{|l|}{ Amen et al. (2007b) } \\
\hline Brahman & 133 & 311.5 & & & 356.1 & 3.8 & 71.7 \\
\hline Angus & 128 & 320.8 & & & 451.4 & 3.3 & 79.4 \\
\hline Difference & & -9.3 NS & & & $-95.3^{*}$ & $0.5 \mathrm{NS}$ & $-7.7^{\star}$ \\
\hline \multicolumn{8}{|l|}{ DeRouen et al. $(1992)^{4}$} \\
\hline Brahman & 216 & 230.0 & & & 3.2 & 13.2 & 62.0 \\
\hline Hereford & 279 & 235.0 & & & 4.1 & 9.9 & 63.0 \\
\hline Difference & & $-5.0^{*}$ & & & $-0.9^{*}$ & $3.3^{*}$ & $-1.0 \mathrm{NS}$ \\
\hline \multicolumn{8}{|l|}{ Baker et al. (2001b) } \\
\hline Brahman & & 334.1 & 3.1 & & 234.5 & 5.3 & 79.1 \\
\hline Hereford & & 320.0 & 3.4 & & 252.3 & 4.4 & 76.7 \\
\hline Difference & & $14.1^{*}$ & $-0.3 \mathrm{NS}$ & & $-17.8 \mathrm{NS}$ & $0.9^{*}$ & 2.4 NS \\
\hline \multicolumn{8}{|l|}{ Wheeler et al. $(2001)^{5}$} \\
\hline Brahman & 119 & 332.0 & 3.3 & 30.0 & 472.9 & 6.0 & 72.9 \\
\hline Hereford & 115 & 345.0 & 3.4 & 72.0 & 522.5 & 4.7 & 73.6 \\
\hline Difference & & $-13.0^{*}$ & $-0.1 \mathrm{NS}$ & $-42.0^{*}$ & $-49.6^{*}$ & $1.3^{*}$ & $-0.7 \mathrm{NS}$ \\
\hline \multicolumn{8}{|l|}{ DeRouen et al. $(1992)^{4}$} \\
\hline Brahman & 216 & 230.0 & & & 3.2 & 13.2 & 62.0 \\
\hline Charolais & 403 & 298.0 & & & 3.9 & 9.5 & 84.0 \\
\hline Difference & & $-68.0^{*}$ & & & $-0.7^{*}$ & $3.7^{*}$ & $-22.0^{*}$ \\
\hline \multicolumn{8}{|c|}{ Brahman vs. other B. indicus sire breeds } \\
\hline Brahman & 128 & 308.2 & 3.7 & 8.8 & 9.4 & 3.9 & 70.5 \\
\hline Sahiwal & 141 & 284.7 & 3.5 & 9.1 & 9.8 & 4.3 & 69.0 \\
\hline Difference & & $23.5^{*}$ & $0.2 \mathrm{NS}$ & $-0.3 \mathrm{NS}$ & $-0.4 \mathrm{NS}$ & $-0.4 \mathrm{NS}$ & $\begin{array}{l}1.5 \mathrm{NS} \\
\text { Continued }\end{array}$ \\
\hline
\end{tabular}




\section{Table 3 (Continued). Carcass trait comparisons involving progeny sired by subtropically adapted beef sire breeds}

\begin{tabular}{|c|c|c|c|c|c|c|c|}
\hline \multirow[b]{2}{*}{ Sire breed comparison } & \multicolumn{7}{|c|}{ Trait $^{1}$} \\
\hline & $\mathbf{N}$ & HCW, kg & YG & QG & MBS & WBS, kg & $\mathrm{M}$ area, $\mathrm{cm}^{2}$ \\
\hline \multicolumn{8}{|l|}{ Crouse et al. (1989) } \\
\hline Brahman & 84 & 285.0 & & & 350.0 & 5.9 & 69.5 \\
\hline Sahiwal & 88 & 258.0 & & & 355.0 & 6.9 & 67.3 \\
\hline Difference & & $27.0^{*}$ & & & $-5.0 \mathrm{NS}$ & $-1.0^{*}$ & $2.2 \mathrm{NS}$ \\
\hline \multicolumn{8}{|l|}{ Paschal et al. (1995) } \\
\hline Gray Brahman & 19 & 300.1 & 2.8 & 344.2 & 347.7 & & 75.5 \\
\hline Red Brahman & 21 & 307.4 & 2.7 & 347.0 & 345.5 & & 77.0 \\
\hline Difference & & $-7.3 \mathrm{NS}$ & $0.1 \mathrm{NS}$ & $-2.8 \mathrm{NS}$ & $2.2 \mathrm{NS}$ & & $-1.5 \mathrm{NS}$ \\
\hline \multicolumn{8}{|l|}{ Paschal et al. (1995) } \\
\hline Gray Brahman & 19 & 300.1 & 2.8 & 344.2 & 347.7 & & 75.5 \\
\hline Gir & 27 & 287.6 & 2.7 & 345.5 & 349.6 & & 77.1 \\
\hline Difference & & $12.5 \mathrm{NS}$ & $0.1 \mathrm{NS}$ & $-1.3 \mathrm{NS}$ & -1.9 NS & & $-1.6 \mathrm{NS}$ \\
\hline \multicolumn{8}{|l|}{ Paschal et al. (1995) } \\
\hline Gray Brahman & 19 & 300.1 & 2.8 & 344.2 & 347.7 & & 75.5 \\
\hline Indu-Brazil & 19 & 287.4 & 2.3 & 344.0 & 344.5 & & 77.5 \\
\hline Difference & & 12.7 NS & $0.5^{*}$ & $0.2 \mathrm{NS}$ & $3.2 \mathrm{NS}$ & & $-2.0 \mathrm{NS}$ \\
\hline \multicolumn{8}{|l|}{ Paschal et al. (1995) } \\
\hline Gray Brahman & 19 & 300.1 & 2.8 & 344.2 & 347.7 & & 75.5 \\
\hline Nellore & 20 & 292.2 & 2.7 & 354.1 & 358.9 & & 75.5 \\
\hline Difference & & $7.9 \mathrm{NS}$ & $0.1 \mathrm{NS}$ & -9.9 NS & $-11.2 \mathrm{NS}$ & & $0.0 \mathrm{NS}$ \\
\hline \multicolumn{8}{|l|}{ Herring et al. (1996) } \\
\hline Brahman & 40 & 311.4 & 3.2 & & 323.9 & 3.6 & 74.9 \\
\hline Boran & 59 & 273.2 & 3.1 & & 344.5 & 3.8 & 72.5 \\
\hline Difference & & $38.2^{*}$ & $0.1 \mathrm{NS}$ & & -20.6 NS & $-0.2 \mathrm{NS}$ & 2.4 NS \\
\hline \multicolumn{8}{|l|}{ Wheeler et al. $(2001)^{5}$} \\
\hline Brahman & 119 & 332.0 & 3.3 & 30.0 & 472.9 & 6.0 & 72.9 \\
\hline Boran & 151 & 310.0 & 3.1 & 47.0 & 503.3 & 5.1 & 74.0 \\
\hline Difference & & $22.0^{*}$ & $0.2 \mathrm{NS}$ & $-17.0^{*}$ & $-30.4^{*}$ & $0.9^{*}$ & $-1.1 \mathrm{NS}$ \\
\hline
\end{tabular}

Brahman vs. non- $B$. indicus subtropically adapted sire breeds

Sanders and Paschal (1987) $)^{2,3}$

Zebu

Senepol

Difference

Herring et al. (1996)

Brahman

Tuli

Difference

Baker et al. (2001b)

Brahman

Tuli

Difference

Wheeler et al. $(2001)^{5}$

Brahman

Tuli

Difference

$\begin{array}{ccccccc}79 & 297.0 & 2.6 & \mathrm{Gd} & \mathrm{SI} & & 75.9 \\ 14 & 281.6 & 2.8 & \mathrm{Gd} & \mathrm{SI} & & 72.3 \\ & 15.4 \mathrm{NT} & -0.2 \mathrm{NT} & -\mathrm{NT} & -\mathrm{NT} & & 3.6 \mathrm{NT} \\ & & & & & & \\ 40 & 311.4 & 3.2 & & 323.9 & 3.6 & 74.9 \\ 43 & 276.2 & 3.0 & & 351.0 & 3.3 & 74.3 \\ & 35.2^{*} & 0.2 \mathrm{NS} & & -27.1^{*} & 0.3 \mathrm{NS} & 0.6 \mathrm{NS} \\ & & & & & & \\ & 334.1 & 3.1 & & 234.5 & 5.3 & 79.1 \\ & 304.2 & 3.0 & & 257.6 & 4.2 & 78.8 \\ & 29.9^{*} & 0.1 \mathrm{NS} & & -23.1^{*} & 1.1^{*} & 0.3 \mathrm{NS} \\ & & & & & & \\ 119 & 332.0 & 3.3 & 30.0 & 472.9 & 6.0 & 72.9 \\ 162 & 308.0 & 3.0 & 63.0 & 523.6 & 4.6 & 73.6 \\ & 24.0^{*} & 0.3^{*} & -33.0^{*} & -50.7^{*} & 1.4^{*} & -0.7 \mathrm{NS}\end{array}$

$B$. indicus-derivative vs. traditional $B$. taurus sire breeds

DeRouen et al. (2000), Louisiana feedlot

Brangus

Angus

Difference

DeRouen et al. (2000), Oklahoma feedlot

$\begin{array}{lllllll}27 & 342.0 & 3.4 & 10.2 & 435.0 & 4.3 & 73.2 \\ 30 & 360.0 & 3.3 & 11.4 & 503.0 & 4.1 & 74.3 \\ & -18.0 \mathrm{NS} & 0.1 \mathrm{NS} & -1.2^{*} & -68.0^{*} & 0.2 \mathrm{NS} & -1.1 \mathrm{NS}\end{array}$




\section{Table 3 (Continued). Carcass trait comparisons involving progeny sired by subtropically adapted beef sire breeds}

\begin{tabular}{|c|c|c|c|c|c|c|c|}
\hline \multirow[b]{2}{*}{ Sire breed comparison } & \multicolumn{7}{|c|}{ Trait $^{1}$} \\
\hline & \multirow{2}{*}{$\frac{\mathbf{N}}{39}$} & \multirow{2}{*}{$\begin{array}{l}\text { HCW, kg } \\
358.0\end{array}$} & \multirow{2}{*}{$\frac{\text { YG }}{3.0}$} & \multirow{2}{*}{$\begin{array}{r}\text { QG } \\
11.2\end{array}$} & \multirow{2}{*}{$\begin{array}{c}\text { MBS } \\
497.0\end{array}$} & \multicolumn{2}{|c|}{ WBS, $\mathrm{kg}$ LM area, $\mathrm{cm}^{2}$} \\
\hline Brangus & & & & & & 4.9 & 79.9 \\
\hline Angus & 30 & 358.0 & 3.1 & 11.5 & 522.0 & 4.6 & 81.7 \\
\hline Difference & & $0.0 \mathrm{NS}$ & $-0.1 \mathrm{NS}$ & $-0.3 \mathrm{NS}$ & $-25.0 \mathrm{NS}$ & $0.3 \mathrm{NS}$ & $-1.8 \mathrm{NS}$ \\
\hline \multicolumn{8}{|l|}{ Bidner et al. (2002) } \\
\hline Brangus & 25 & 340.0 & 3.3 & 11.9 & 560.0 & 4.7 & 73.8 \\
\hline Angus & 48 & 290.0 & 3.1 & 11.5 & 510.0 & 4.0 & 70.4 \\
\hline Difference & & $50.0^{*}$ & $0.2 \mathrm{NS}$ & $0.4 \mathrm{NS}$ & $50.0 \mathrm{NS}$ & $0.7 \mathrm{NS}$ & $3.4 \mathrm{NS}$ \\
\hline \multicolumn{8}{|l|}{ Wheeler et al. $(2006)^{5}$} \\
\hline Brangus & 107 & 351.1 & 2.7 & 47.4 & 497.0 & 3.9 & 85.7 \\
\hline Angus & 103 & 355.2 & 3.2 & 68.9 & 548.0 & 3.4 & 83.0 \\
\hline Difference & & $-4.1 \mathrm{NS}$ & $-0.5^{*}$ & $-21.5^{*}$ & $-51.0^{*}$ & $0.5^{*}$ & $2.7^{*}$ \\
\hline \multicolumn{8}{|l|}{ Wheeler et al. $(2006)^{5}$} \\
\hline Brangus & 107 & 351.1 & 2.7 & 47.4 & 497.0 & 3.9 & 85.7 \\
\hline Hereford & 102 & 343.4 & 2.9 & 51.6 & 515.0 & 3.7 & 81.7 \\
\hline Difference & & $7.7 \mathrm{NS}$ & $-0.2 \mathrm{NS}$ & $-4.2 \mathrm{NS}$ & -18.0 NS & $0.2 \mathrm{NS}$ & $4.0^{*}$ \\
\hline \multicolumn{8}{|c|}{ DeRouen et al. (2000), Louisiana feedlot } \\
\hline Brangus & 27 & 342.0 & 3.4 & 11.2 & 435.0 & 4.3 & 73.2 \\
\hline Gelbvieh & 26 & 371.0 & 2.8 & 10.7 & 443.0 & 4.9 & 88.4 \\
\hline Difference & & $-29.0^{*}$ & $0.6 \mathrm{NS}$ & $0.5 \mathrm{NS}$ & $-8.0 \mathrm{NS}$ & $-0.6 \mathrm{NS}$ & -15.2 NS \\
\hline \multicolumn{8}{|c|}{ DeRouen et al. (2000), Oklahoma feedlot } \\
\hline Brangus & 39 & 358.0 & 3.0 & 11.2 & 497.0 & 4.9 & 79.9 \\
\hline Gelbvieh & 23 & 353.0 & 2.4 & 10.7 & 456.0 & 5.3 & 87.5 \\
\hline Difference & & $5.0 \mathrm{NS}$ & $0.6 \mathrm{NS}$ & $0.5 \mathrm{NS}$ & $41.0 \mathrm{NS}$ & $-0.4 \mathrm{NS}$ & $-7.6 \mathrm{NS}$ \\
\hline \multicolumn{8}{|c|}{ DeRouen et al. (2000), Louisiana feedlot } \\
\hline Gelbray & 31 & 332.0 & 2.9 & 10.5 & 443.0 & 4.8 & 72.3 \\
\hline Angus & 30 & 360.0 & 3.3 & 11.4 & 503.0 & 4.1 & 74.3 \\
\hline Difference & & $-28.0^{*}$ & $-0.4 \mathrm{NS}$ & $-0.9 \mathrm{NS}$ & $-60.0^{*}$ & $0.7 \mathrm{NS}$ & $-2.0 \mathrm{NS}$ \\
\hline \multicolumn{8}{|c|}{ DeRouen et al. (2000), Oklahoma feedlot } \\
\hline Gelbray & 25 & 344.0 & 2.8 & 10.8 & 460.0 & 5.0 & 80.0 \\
\hline Angus & 30 & 358.0 & 3.1 & 11.5 & 522.0 & 4.6 & 81.7 \\
\hline Difference & & $-14.0 \mathrm{NS}$ & $-0.3 \mathrm{NS}$ & $-0.7 \mathrm{NS}$ & $-62.0^{*}$ & $0.4 \mathrm{NS}$ & $-1.7 \mathrm{NS}$ \\
\hline \multicolumn{8}{|l|}{ Bidner et al. (2002) } \\
\hline Gelbray & 19 & 354.0 & 2.5 & 10.8 & 461.0 & 4.7 & 86.7 \\
\hline Angus & 48 & 290.0 & 3.1 & 11.5 & 510.0 & 4.0 & 70.4 \\
\hline Difference & & $64.0^{*}$ & -0.6 NS & $-0.7 \mathrm{NS}$ & $-49.0 \mathrm{NS}$ & $0.7 \mathrm{NS}$ & $16.3^{*}$ \\
\hline \multicolumn{8}{|c|}{ DeRouen et al. (2000), Louisiana feedlot } \\
\hline Gelbray & 31 & 332.0 & 2.9 & 10.5 & 443.0 & 4.8 & 72.3 \\
\hline Gelbvieh & 26 & 371.0 & 2.8 & 10.7 & 443.0 & 4.9 & 88.4 \\
\hline Difference & & $-39.0^{*}$ & $0.1 \mathrm{NS}$ & $-0.2 \mathrm{NS}$ & $0.0 \mathrm{NS}$ & $-0.1 \mathrm{NS}$ & $-16.1 \mathrm{NS}$ \\
\hline DeRouen et al. (2000), C & & & & & & & \\
\hline Gelbray & 25 & 344.0 & 2.8 & 10.8 & 460.0 & 5.0 & 80.0 \\
\hline Gelbvieh & 23 & 353.0 & 2.4 & 10.7 & 456.0 & 5.3 & 87.5 \\
\hline Difference & & $-9.0 \mathrm{NS}$ & $0.4 \mathrm{NS}$ & $0.1 \mathrm{NS}$ & $4.0 \mathrm{NS}$ & $-0.3 \mathrm{NS}$ & $-7.5 \mathrm{NS}$ \\
\hline Bidner et al. (2002) & & & & & & & \\
\hline Beefmaster & 34 & 313.0 & 3.3 & 10.4 & 445.0 & 5.1 & 70.0 \\
\hline Angus & 48 & 290.0 & 3.1 & 11.5 & 510.0 & 4.0 & 70.4 \\
\hline Difference & & $23.0 \mathrm{NS}$ & $0.2 \mathrm{NS}$ & $-1.1 \mathrm{NS}$ & $-65.0 \mathrm{NS}$ & $1.1 \mathrm{NS}$ & $-0.4 \mathrm{NS}$ \\
\hline Wheeler et al. $(2006)^{5}$ & & & & & & & \\
\hline Beefmaster & 103 & 357.9 & 3.1 & 32.1 & 483.0 & 4.1 & 82.3 \\
\hline Angus & 103 & 355.2 & 3.2 & 68.9 & 548.0 & 3.4 & 83.0 \\
\hline Difference & & $2.7 \mathrm{NS}$ & $-0.1 \mathrm{NS}$ & $-36.8^{*}$ & $-65.0^{*}$ & $0.7^{*}$ & $-0.7 \mathrm{NS}$ \\
\hline Wheeler et al. $(2006)^{5}$ & & & & & & & \\
\hline Beefmaster & 103 & 357.9 & 3.1 & 32.1 & 483.0 & 4.1 & 82.3 \\
\hline Hereford & 102 & 343.4 & 2.9 & 51.6 & 515.0 & 3.7 & 81.7 \\
\hline
\end{tabular}




\section{Table 3 (Continued). Carcass trait comparisons involving progeny sired by subtropically adapted beef sire breeds}

\begin{tabular}{|c|c|c|c|c|c|c|c|}
\hline \multirow[b]{2}{*}{ Sire breed comparison } & \multicolumn{7}{|c|}{ Trait $^{1}$} \\
\hline & \multirow[t]{2}{*}{$\mathbf{N}$} & \multirow{2}{*}{$\frac{\mathrm{HCW}, \mathbf{k g}}{14.5^{*}}$} & \multirow{2}{*}{$\frac{\text { YG }}{0.2 \mathrm{NS}}$} & \multirow{2}{*}{$\frac{\mathbf{Q G}}{-19.5^{*}}$} & \multirow{2}{*}{$\begin{array}{r}\text { MBS } \\
-32.0^{*}\end{array}$} & \multicolumn{2}{|c|}{ WBS, $\mathrm{kg}$ LM area, $\mathrm{cm}^{2}$} \\
\hline Difference & & & & & & $0.4^{*}$ & $0.6 \mathrm{NS}$ \\
\hline \multicolumn{8}{|l|}{ Bidner et al. (2002) } \\
\hline Simbrah & 31 & 388.0 & 3.0 & 11.0 & 483.0 & 4.4 & 86.2 \\
\hline Angus & 48 & 290.0 & 3.1 & 11.5 & 510.0 & 4.0 & 70.4 \\
\hline Difference & & $98.0^{*}$ & $-0.1 \mathrm{NS}$ & $-0.5 \mathrm{NS}$ & $-27.0 \mathrm{NS}$ & $0.4 \mathrm{NS}$ & $15.8^{*}$ \\
\hline \multicolumn{8}{|c|}{$\begin{array}{l}\text { B. indicus-derivative vs. non-B. indicus subtropically } \\
\text { adapted sire breeds } \\
\text { Wheeler et al. }(2006)^{5}\end{array}$} \\
\hline Brangus & 107 & 351.1 & 2.7 & 47.4 & 497.0 & 3.9 & 85.7 \\
\hline Romosinuano & 100 & 318.4 & 2.3 & 33.7 & 488.0 & 3.8 & 83.6 \\
\hline Difference & & $32.7^{*}$ & $0.4 \mathrm{NS}$ & 13.7 NS & $9.0 \mathrm{NS}$ & $0.1 \mathrm{NS}$ & $2.1 \mathrm{NS}$ \\
\hline \multicolumn{8}{|l|}{ Wheeler et al. $(2006)^{5}$} \\
\hline Brangus & 107 & 351.1 & 2.7 & 47.4 & 497.0 & 3.9 & 85.7 \\
\hline Bonsmara & 104 & 330.7 & 2.4 & 38.9 & 487.0 & 3.7 & 85.9 \\
\hline Difference & & $20.4^{*}$ & $0.3^{*}$ & $8.5 \mathrm{NS}$ & 10.0 NS & $0.2^{*}$ & $-0.2 \mathrm{NS}$ \\
\hline \multicolumn{8}{|l|}{ Wheeler et al. $(2006)^{5}$} \\
\hline Beefmaster & 103 & 357.9 & 3.1 & 32.1 & 483.0 & 4.1 & 82.3 \\
\hline Romosinuano & 102 & 318.4 & 2.3 & 33.7 & 488.0 & 3.8 & 83.6 \\
\hline Difference & & $39.5^{*}$ & $0.8^{*}$ & $-1.6 \mathrm{NS}$ & $-5.0 \mathrm{NS}$ & $0.3^{*}$ & $-1.3 \mathrm{NS}$ \\
\hline \multicolumn{8}{|l|}{ Wheeler et al. $(2006)^{5}$} \\
\hline Beefmaster & 103 & 357.9 & 3.1 & 32.1 & 483.0 & 4.1 & 82.3 \\
\hline Bonsmara & 104 & 330.7 & 2.4 & 38.9 & 487.0 & 3.7 & 85.9 \\
\hline Difference & & $27.2^{*}$ & $0.7^{*}$ & $-6.8 \mathrm{NS}$ & $-4.0 \mathrm{NS}$ & $0.4^{*}$ & $-3.6^{*}$ \\
\hline
\end{tabular}

Non- $B$. indicus subtropically adapted vs. traditional $B$. taurus sire breeds

Baker et al. (2001b)

Tuli

Angus

Difference

Baker et al. (2001b)

Tuli

Hereford

Difference

Sanders and Paschal (1987) ${ }^{3}$

Senepol

Angus

Difference

Chase et al. (1998), phase II

Senepol

Hereford

Difference

Wheeler et al. (2006)

Romosinuano

Angus

Difference

Wheeler et al. (2006)

Romosinuano

Hereford

Difference

Phillips et al. (2006)

Romosinuano

Angus

$\begin{array}{lc}304.2 & 3.0 \\ 332.7 & 3.4 \\ -28.5^{*} & -0.4^{*} \\ & \\ 304.2 & 3.0 \\ 320.0 & 3.4 \\ -15.8^{*} & -0.4^{*}\end{array}$

$\begin{array}{lll}14 & 281.6 & 2.8\end{array}$

$\begin{array}{lll}28 & 285.4 & 2.6\end{array}$

$-3.8 \mathrm{NT}$

$0.2 \mathrm{NT}$

56

62

261.0

256.5

$4.5 \mathrm{NS}$

2.7

2.6

$0.1 \mathrm{NS}$

102

103

318.4

$355.2 \quad 3.2$

$-36.8^{*}-0.9^{*}$

$100 \quad 318.4$

102

318.4
343.4

343.4

-25.0 *

$$
2.3
$$

2.9

$-0.6^{*}$

$99 \quad 298.0$

$51 \quad 290.0$
257.6

269.4

-11.8 NS

4.2

4.0

0.2 NS

257.

252.3

4.2
4.4

$5.3 \mathrm{NS}-0.2 \mathrm{NS}$

76.7

2.1 NS

72.3

75.8

-3.5 NT

- NT

Sm

- NT

69.9

566.0

369.5

4.9

4.6

70.4

-4.5 NS

375.5

$0.3 \mathrm{NS}$

$-0.5 \mathrm{NS}$

83.6

\section{7}

68.9

$-35.2^{*}$

488.0

3.8

3.4

$0.4^{*}$

83.0

0.6 NS

\section{7}

$-60.0^{*}$

3.8

3.7

83.6

81.7

1.9 NS

$-17.9^{*}$

$-27.0^{*}$

$0.1^{*}$

75.2

72.5

Continued 
Table 3 (Continued). Carcass trait comparisons involving progeny sired by subtropically adapted beef sire breeds

\begin{tabular}{|c|c|c|c|c|c|c|c|}
\hline \multirow[b]{2}{*}{ Sire breed comparison } & \multicolumn{7}{|c|}{ Trait $^{1}$} \\
\hline & $\mathbf{N}$ & HCW, kg & YG & QG & MBS & WBS, $\mathbf{k g}$ & LM area, $\mathbf{c m}^{2}$ \\
\hline Difference & & $8.0 \mathrm{NS}$ & $-0.3^{\star *}$ & $-1.4^{\star *}$ & $-0.9^{* *}$ & & $2.7^{*}$ \\
\hline \multicolumn{8}{|l|}{ Wheeler et al. $(2006)^{5}$} \\
\hline Bonsmara & 104 & 330.7 & 2.4 & 38.9 & 487.0 & 3.7 & 85.9 \\
\hline Angus & 103 & 355.2 & 3.2 & 68.9 & 548.0 & 3.4 & 83.0 \\
\hline Difference & & $-24.5^{*}$ & $-0.8 \mathrm{NS}$ & $-30.0^{*}$ & $-61.0^{*}$ & $0.3^{*}$ & $2.9^{*}$ \\
\hline \multicolumn{8}{|l|}{ Wheeler et al. $(2006)^{5}$} \\
\hline Bonsmara & 104 & 330.7 & 2.4 & 38.9 & 487.0 & 3.7 & 85.9 \\
\hline Hereford & 102 & 343.4 & 2.9 & 51.6 & 515.0 & 3.7 & 81.7 \\
\hline Difference & & $-12.7^{*}$ & $-0.5^{*}$ & $-12.7 \mathrm{NS}$ & $-28.0^{*}$ & $0.0 \mathrm{NS}$ & $4.2^{*}$ \\
\hline
\end{tabular}

${ }^{1} \mathrm{HCW}=$ hot carcass weight; MBS = marbling score; WBS = Warner-Bratzler shear force value.

${ }^{2}$ Zebu collectively refers to average for Gray Brahman, Red Brahman, Gir, Indu-Brazil, and Nellore.

${ }^{3} \mathrm{Gd}=$ Good; $\mathrm{Ch}=$ Choice; SI = Slight; Sm = Small.

${ }^{4} \mathrm{~N}$ values taken from Franke et al. (2001).

${ }^{5} \mathrm{QG}$ refers to percentage of Choice carcasses.

${ }^{\star *} P<0.01 ;{ }^{*} P<0.05$; NS $=$ not significant $(P>0.10)$; NT $=$ not tested.

and Hereford-sired progeny express higher marbling scores, higher QG, and lower Warner-Bratzler shear force values (indicating less carcass tenderness for Brahman-sired progeny). The lack of carcass tenderness expressed by Brahman-influenced cattle has been addressed previously (Thrift and Thrift, 2002).

Carcass YG and LM area tend to be similar for Brahman-, Angus-, and Hereford-sired progeny. With the exception of 2 studies (Paschal et al., 1995; Baker et al., 2001b), most studies have indicated similar or greater carcass weights for Angus-, Hereford-, or Charolais-sired progeny relative to Brahman-sired progeny.

Brahman Versus Other Bos indicus Sire Breeds. Brahman-sired progeny have greater carcass weights than Sahiwal- and Boran-sired progeny; however, carcass weight differences between Gray Brahman- and other B. indicus-sired progeny (Red Brahman, Gir, Indu-Brazil, Nellore) are small. All other carcass trait differences between Brahman and other B. indicus sire breeds appear small, although Wheeler et al. (2001) indicated advantages for Boran-sired progeny in terms of QG, marbling score, and Warner-Bratzler shear force value. These latter advantages for the Boran were not evident in the study by Herring et al. (1996).

Brahman Versus non-B. indicus Subtropically Adapted Sire Breeds. Brahman-sired progeny tend to have heavier carcass weights, lower marbling scores, similar or slightly higher Warner-Bratzler shear force values, and similar YG and LM area compared with Tuli-sired progeny. The slightly higher Warner-Bratzler shear force values for Brahman-sired progeny indicate a slight advantage for carcass tenderness of Tuli-sired progeny.

Bos indicus-Derivative Versus Traditional Bos taurus Sire Breeds. Relative to Angus- and Hereford-sired progeny, Brangus- and Beefmaster-sired progeny tend to have similar or lower marbling scores and QG. Overall, carcass weight, YG, Warner-Bratzler shear force value, and LM area tend to be similar for progeny of $B$. indicus-derivative and traditional B. taurus sire breeds. Carcass traits tend to be similar for Brangus- and Gelbvieh-sired progeny. With regard to marbling score, Gelbray- and Gelbvieh-sired progeny are similar; however, Gelbray-sired progeny have lower marbling scores than Angus-sired progeny.

Bos indicus-Derivative Versus non-Bos indicus Subtropically Adapted Sire Breeds. Brangus- and Beefmaster-sired progeny have greater carcass weights and higher WarnerBratzler shear force values (indicating less carcass tenderness) than Romosinuano- and Bonsmara-sired progeny. Other carcass trait differences for these sire breed genetic types appear to be small.

Non-Bos indicus Subtropically Adapted Versus Traditional Bos taurus Sire Breeds. Tuli-, Romosinuano-, and Bonsmara-sired progeny have lighter carcass weights, lower marbling scores (especially true for Romosinuano and Bonsmara), and lower QG than Angus- or Herefordsired progeny. Warner-Bratzler shear force values are similar for Tuli-, Angus-, and Hereford-sired progeny; however, Bonsmara- and Romosinuano-sired progeny tend to have higher Warner-Bratzler shear force values than Angus- or Hereford-sired progeny, indicating no advantage in carcass tenderness for these non- $B$. indicus subtropically adapted genetic types. 
Carcass traits appear to be similar for Senepol-, Angus-, and Hereford-sired progeny.

\section{IMPLICATIONS}

Evaluation of several non- $B$. indicus subtropically adapted sire breeds suggests that some of these sire breeds may serve as alternatives to some $B$. indicus genetic types because of their ability to tolerate hot, humid conditions in the US Southeast and Gulf Coast areas. Specifically, considering Brahman as the standard B. indicus sire breed, results of this review indicate the non- $B$. indicus subtropically adapted sire breeds will contribute to less dystocia but are expected to sire progeny that weigh less at weaning, grow at a slower rate postweaning, and have lighter carcasses than Brahman-sired progeny. Further, progeny of non- $B$. indicus subtropically adapted sire breeds are expected to have slightly improved carcass merit, especially in regard to carcass tenderness, relative to Brahman-sired progeny. However, there appears to be no advantage in carcass merit for progeny of non- $B$. indicus subtropically adapted sire breeds relative to traditional $B$. taurus sire breeds such as the Angus and Hereford. Overall, the suitability of the non- $B$. indicus subtropically adapted sire breeds, as potential alternatives for $B$. indicus genetic types, can be determined only after maternal performance of $\mathrm{F}_{1}$ females has been assessed. In addition, it is important to determine if price discounts, similar to those applied to $B$. indicus-influenced cattle, will be incurred for progeny of non- $B$. indicus subtropically adapted sire breeds when marketing occurs through traditional channels.

\section{LITERATURE CITED}

Amen, T. S., A. D. Herring, J. O. Sanders, and C. A. Gill. 2007a. Evaluation of reciprocal differences in Bos indicus $\times$ Bos taurus backcross calves produced through embryo transfer: I. Birth and weaning traits. J. Anim. Sci. 85:365.

Amen, T. S., A. D. Herring, J. O. Sanders, and C. A. Gill. 2007b. Evaluation of recipro- cal differences in Bos indicus $\times$ Bos taurus backcross calves produced through embryo transfer: II. Postweaning, carcass, and meat traits. J. Anim. Sci. 85:373.

Baker, J. F., S. V. Tucker, and R. C. Vann. 2001a. Effects of Tuli, Senepol, Brahman, Angus, and Polled Hereford sire breeds on birth and weaning traits of offspring. Prof. Anim. Sci. 17:160.

Baker, J. F., S. E. Williams, and R. C. Vann. 2001b. Effects of Tuli, Brahman, Angus, and Polled Hereford sires on carcass traits of steer offspring. Prof. Anim. Sci. 17:154.

Bidner, T. D., W. E. Wyatt, P. E. Humes, D. E. Franke, and D. C. Blouin. 2002. Influence of Brahman-derivative breeds and Angus on carcass traits, physical composition, and palatability. J. Anim. Sci. 80:2126.

Brown, M. A., A. H. Brown Jr., W. G. Jackson, and J. R. Miesner. 1993a. Genotype $\times$ environment interactions in postweaning performance to yearling in Angus, Brahman, and reciprocal-cross calves. J. Anim. Sci. 71:3273

Brown, M. A., A. H. Brown Jr., W. G. Jackson, and J. R. Miesner. 1997. Preweaning performance of Beefmaster- and Brahmansired calves from Angus and Brahman cows on three forage systems. Prof. Anim. Sci. 13:90.

Brown, M. A., and D. L. Lalman. 2008. Preweaning performance of calves from Bonsmara, Brangus, Charolais, Gelbvieh, Hereford, and Romosinuano sires bred to Brangus cows managed on native rangeland or improved forages. Prof. Anim. Sci. 24:67.

Brown, M. A., W. A. Phillips, A. H. Brown Jr., S. W. Coleman, W. G. Jackson, and J. R. Miesner. 1999. Postweaning performance of calves from Angus, Brahman, and reciprocalcross cows grazing endophyte-infected tall fescue or common bermudagrass. J. Anim. Sci. $77: 25$.

Brown, M. A., L. M. Tharel, A. H. Brown Jr., W. G. Jackson, and J. R. Miesner. 1993b. Genotype $\times$ environment interactions in preweaning traits of purebred and reciprocal cross Angus and Brahman calves on common bermudagrass and endophyte-infected tall fescue pastures. J. Anim. Sci. 71:326.

Brown, M. A., X. Z. Wang, F. Q. Gao, J. P. Wu, D. L. Lalman, and J. W. Holloway. 2008. Postweaning gains in calves sired by six sire breeds evaluated on two preweaning forages and two postweaning management systems. Prof. Anim. Sci. 24:224.

Browning, R., Jr., M. L. Leite-Browning, D. A. Neuendorff, and R. D. Randel. 1995. Preweaning growth of Angus- (Bos taurus), Brahman- (Bos indicus), and Tuli- (Sanga) sired calves and reproductive performance of their Brahman dams. J. Anim. Sci. 73:2558.

Chase, C. C., Jr., A. C. Hammond, and T. A. Olson. 2000. Effect of tropically adapted sire breeds on preweaning growth of $\mathrm{F}_{1}$ Angus calves and reproductive performance of their Angus dams. J. Anim. Sci. 78:1111.

Chase, C. C., Jr., T. A. Olson, A. C. Hammond, M. A. Menchaca, R. L. West, D. D. Johnson, and W. T. Butts Jr. 1998. Preweaning growth traits for Senepol, Hereford, and reciprocal crossbred calves and feedlot performance and carcass characteristics of steers. J. Anim. Sci. 76:2967.

Crouse, J. D., L. V. Cundiff, R. M. Koch, M. Koohmaraie, and S. C. Seideman. 1989. Comparisons of Bos indicus and Bos taurus inheritance for carcass beef characteristics and meat palatability. J. Anim. Sci. 67:2661.

Cundiff, L. V. 2005. Performance of tropically adapted breeds in a temperate environment: Calving, growth, reproduction and maternal traits. p. 131 in A Compilation of Research Results Involving Tropically Adapted Beef Cattle Breeds. Southern Coop. Ser. Bull. 405. http://www.lsuagcenter.com/en/crops_livestock/livestock/beef_cattle/breeding_genetics/tropical+breeds.htm Accessed Dec. 16, 2009 .

DeRouen, S. M., D. E. Franke, T. D. Bidner, and D. C. Blouin. 1992. Two-, three-, and four-breed rotational crossbreeding of beef cattle: Carcass traits . J. Anim. Sci. 70:3665.

DeRouen, S. M., W. E. Wyatt, T. D. Bidner, and M. A. Persica. 2000. Feedlot and carcass performance of Angus-, Brangus-, Gelbvieh-, and Gelbray-sired crossbred steers. Prof. Anim. Sci. 16:6.

Franke, D. E. 1980. Breed and heterosis effects of American Zebu cattle. J. Anim. Sci. 50:1206.

Franke, D. E., O. Habet, L. C. Tawah, A. R. Williams, and S. M. DeRouen. 2001. Direct and maternal genetic effects on birth and weaning traits in multibreed cattle data and predicted performance of breed crosses. J. Anim. Sci. 79:1713

Gregory, K. E., and L. V. Cundiff. 1980. Crossbreeding in beef cattle: Evaluation of systems. J. Anim. Sci. 51:1224.

Gregory, K. E., G. M. Smith, L. V. Cundiff, R. M. Koch, and D. B. Laster. 1979. Characterization of biological types of cattle - Cycle III: I. Birth and weaning traits. J. Anim. Sci. $48: 271$

Herring, A. D., J. O. Sanders, R. E. Knutson, and D. K. Lunt. 1996. Evaluation of $F_{1}$ calves sired by Brahman, Boran and Tuli bulls for birth, growth, size and carcass characteristics. J. Anim. Sci. 74:955.

Holloway, J. W., B. G. Warrington, D. W. Forrest, and R. D. Randel. 2002. Preweaning growth of $\mathrm{F}_{1}$ tropically adapted beef cattle breeds $\times$ Angus and reproductive performance of their Angus dams in arid rangeland. J. Anim. Sci. 80:911.

Koch, R. M., M. E. Dikeman, and J. D. Crouse. 1982. Characterization of biological types of cattle (Cycle III). III. Carcass com- 
position, quality and palatability. J. Anim. Sci. 54:35.

Koger, M. 1980. Effective crossbreeding systems utilizing Zebu cattle. J. Anim. Sci. 50:1215.

Long, C. R. 1980. Crossbreeding for beef production: Experimental results. J. Anim. Sci. 51:1197.

Neville, W. E. Jr., S. V. Tucker, and P. R. Utley. 1988. Reproduction and calf performance of Angus-sired and Polled Herefordsired cows bred to Angus and Brahman bulls for primiparous calving as two- vs three-yearolds. J. Anim. Sci. 66:1606.

Oxford, E. L., A. H. Brown Jr., Z. B. Johnson, and D. W. Kellogg. 2006. Case Study: Sire breed effects on preweaning traits of crossbred and purebred calves from Angus or Hereford dams. Prof. Anim. Sci. 22:59.

Paschal, J. C., J. O. Sanders, and J. L. Kerr. 1991. Calving and weaning characteristics of Angus-, Gray Brahman-, Gir-, Indu-Brazil-, Nellore- and Red Brahman-sired $\mathrm{F}_{1}$ calves. J. Anim. Sci. 69:2395.

Paschal, J. C., J. O. Sanders, J. L. Kerr, D. K. Lunt, and A. D. Herring. 1995. Postweaning and feedlot growth and carcass characteristics of Angus-, Gray Brahman-, Gir-, InduBrazil-, Nellore-, and Red Brahman-sired $F_{1}$ calves. J. Anim. Sci. 73:373.

Phillips, W. A., S. W. Coleman, D. G. Riley, C. C. Chase Jr., and H. S. Mayeux. 2006.
Stocker and feedlot performance and carcass characteristics of purebred Angus and Romosinuano steers. Prof. Anim. Sci. 22:8.

Riley, D. G., C. C. Chase Jr., S. W. Coleman, and T. A. Olson. 2007. Evaluation of birth and weaning traits of Romosinuano calves as purebreds and crosses with Brahman and Angus. J. Anim. Sci. 85:289.

Roberson, R. L., J. O. Sanders, and T. C. Cartwright. 1986. Direct and maternal genetic effects on preweaning characters of Brahman, Hereford and Brahman-Hereford crossbred cattle. J. Anim. Sci. 63:438.

Sanders, J. O., and J. C. Paschal. 1987. Postweaning growth and carcass characteristics of Senepol-, Angus- and Zebu-sired crossbred steers. Int. Senepol Res. Symp. 1:123.

Sanders, J. O., J. C. Paschal, and R. M. Thallman. 1987. Preweaning performance of Senepol-, Angus-, and Zebu-sired calves in Texas. Int. Senepol Res. Symp. 1:75.

Thrift, F. A., S. M. Clark, D. K. Aaron, and D. E. Franke. 1999. Interaction between calf sex and calf genetic type for preweaning traits of calves varying in percentage Brahman breeding. Prof. Anim. Sci. 13:145.

Thrift, F. A., D. E. Franke, and D. K. Aaron 1986. Preweaning breed-of-sire comparisons involving the Senepol breed of cattle. J. Anim. Sci. 62:1247.

Thrift, F. A., D. E. Franke, and T. A. Thrift. 2002. Review: The issue of dystocia expressed when sires varying in percent Bos indicus inheritance are mated to Bos taurus females. Prof. Anim. Sci. 18:18.

Thrift, F. A., and T. A. Thrift. 2002. Review: The issue of carcass tenderness expressed by cattle varying in Bos indicus inheritance. Prof. Anim. Sci. 18:193.

Thrift, F. A., and T. A. Thrift. 2005. Rationale for evaluating alternative sources of subtropically adapted beef cattle germplasm. p. 6 in A Compilation of Research Results Involving Tropically Adapted Beef Cattle Breeds. Southern Coop. Ser. Bull. 405. http://www.lsuagcenter.com/en/crops_livestock/livestock/beef_cattle/breeding_genetics/tropical+breeds.htm Accessed Dec. 16, 2009 .

Wheeler, T. L., L. V. Cundiff, S. D. Shackelford, and M. Koohmaraie. 2001. Characterization of biological types of cattle (Cycle V): Carcass traits and longissimus palatability. J. Anim. Sci. 79:1209.

Wheeler, T. L., L. V. Cundiff, L. D. Van Vleck, G. D. Snowder, R. M. Thallman, S. D. Shackelford, and M. Koohmaraie. 2006. Preliminary results from Cycle VIII of the cattle germplasm evaluation program at the Roman L. Hruska US Meat Animal Research Center. Germplasm Eval. Program Prog. Rep. No. 23. Roman L. Hruska US Meat Anim. Res. Cent., Clay Center, NE. 